NBI-HE-94-36

hep-ph/9407329

July, 1994

\title{
Exact Multiparticle Amplitudes at Threshold in $\phi^{4}$ Theories with Softly Broken $O(\infty)$ Symmetry
}

\author{
Minos Axenides' \\ The Niels Bohr Institute, \\ Blegdamsvej 17, 2100 Copenhagen, Denmark \\ Andrei Johansenf \\ St.Petersburg Nuclear Physics Institute, \\ Gatchina, 188350 St.Petersburg, Russian Federation
}

\author{
Yuri Makeenko \\ The Niels Bohr Institute, \\ Blegdamsvej 17, 2100 Copenhagen, Denmark \\ and \\ Institute of Theoretical and Experimental Physics, \\ B. Cheremushkinskaya 25, 117259 Moscow, Russian Federation
}

\footnotetext{
${ }^{1} \mathrm{E}$-mail: axenides@nbivax.nbi.dk

${ }^{2} \mathrm{E}$-mail: ajohansen@nbivax.nbi.dk / johansen@lnpi.spb.su

${ }^{3}$ E-mail: makeenko@nbivax.nbi.dk / makeenko@vxitep.itep.msk.su
} 


\begin{abstract}
We consider the problem of multiparticle production at threshold in a $\phi^{4}$-theory with an $O\left(N_{1}+N_{2}\right)$ symmetry softly broken down to $O\left(N_{1}\right) \times O\left(N_{2}\right)$ by nonequal masses. We derive the set of recurrence relations between the multiparticle amplitudes which sums all relevant diagrams with arbitrary number of loops in the large$N$ limit with fixed number of produced particles. We transform it into a quantum mechanical problem and show how it can be obtained directly from the operator equations of motion by applying the factorization at large $N$. We find the exact solutions to the problem by using the Gelfand-Dikiu representation of the diagonal resolvent of the Schrödinger operator. The result coincides with the tree amplitudes while the effect of loops is the renormalization of the coupling constant and masses. The form of the solution is due to the fact that the exact amplitude of the process $2 \rightarrow n$ vanishes at $n>2$ on mass shell when averaged over the $O\left(N_{1,2}\right)$-indices of incoming particles. We discuss what dynamical symmetry is behind this property. We also give an exact solution in the large- $N$ limit for the model of the $O(N)+$ singlet scalar particle with the spontaneous breaking of a reflection symmetry.
\end{abstract}

Submitted to Nuclear Physics B 


\section{Introduction}

The problem of calculating amplitudes of multiparticle production at threshold has recently received a considerable interest [1]-[27]. The explicit results [6, 7] for the tree level amplitudes in the $\phi^{4}$ theory demonstrate the factorial growth which is expected due to the large number of identical bosons in the final state. While the threshold amplitudes have a vanishing phase volume, they are a lower bound for tree amplitudes with nonvanishing spatial momenta of produced particles which violate unitarity [4, 8, 9]. Therefore, loops should be taken into account.

The one-loop calculation of the $1 \rightarrow n$ amplitude discovered [12] a very interesting property of the tree $2 \rightarrow n$ threshold amplitude which is that it vanishes for $n>4$ when incoming particles are on mass-shell. This nullification has been extended [13] [25] to more general models and is crucial for calculations of the amplitude $1 \rightarrow n$ at the oneloop level. A dynamical symmetry which may be responsible for the nullification has been discussed in Ref. [24]. An interesting question is which properties of the tree and one-loop amplitudes could survive in the full theory which includes all loop diagrams.

The simplest exactly solvable model is the $O(N)$-symmetric $\phi^{4}$ theory in the large- $N$ limit which is solvable [28] since only the bubble diagrams contribute if there is no multiparticle production. In Ref. [26] the set of recurrence relations between the amplitudes of multiparticle production at threshold has been derived in the large- $N$ limit at fixed number of produced particles $n$ which sums all relevant diagrams with an arbitrary number of loops. These recurrence relations have been transformed into a quantum mechanical problem whose exact solution has been found through the use of the Gelfand-Dikil representation of the diagonal resolvent of the Schrödinger operator. The result was quite similar to the tree amplitudes while the effect of loops is the renormalization of the coupling constant and mass. The form of the solution was due to the fact that the exact amplitude of the process $2 \rightarrow n$ vanishes for $n>2$ at large $N$ on mass shell when averaged over the $O(N)$-indices of incoming particles. This nullification occurs for dynamical reasons because of the cancellation between diagrams.

In the present paper we extend the approach of Ref. [26] to more complicated models. We consider the model of two scalar fields: a $N_{1}$-component field with the mass $m_{1}$ and a $N_{2}$-component one with the mass $m_{2}$. The interaction is $O\left(N_{1}+N_{2}\right)$ invariant while the mass term breaks this symmetry down to $O\left(N_{1}\right) \times O\left(N_{2}\right)$ for nonequal masses $m_{1}$ and $m_{2}$. We consider also the case when the second field is a single component one (i.e. $N_{2}=1$ ) and has a wrong sign of the mass square, $m_{2}^{2}<0$, which results in the spontaneous breaking of a reflection symmetry. The motivations for studying this model are as follows:

1. The tree amplitudes $1 \rightarrow n$ at threshold can be found for the model with the softly broken $O(N)$-symmetry extending the results of Ref. [23] given for $N=2$. A question arises as to how these results, obtained exploiting in analogy with 1-dimensional integrable systems, can survive at large $N$ in higher loops. 
2. The model with $N_{2}=1$ and $m_{2}^{2}<0$ describes after the spontaneous breaking of a reflection symmetry 1 particle with mass $\sqrt{2}\left|m_{2}\right|$ and $N-1$ particles with mass $\sqrt{m_{1}^{2}+\left|m_{2}^{2}\right|}$. If the $O(N)$-symmetry were not explicitly broken by masses (i.e. if $\left.m_{1}^{2}=m_{2}^{2}\right)$, these $N-1$ particles would be massless - the Goldstone bosons associated with a spontaneous breaking of the $O(N)$-symmetry — so that the consideration would not be possible. Having the large- $N$ solution, one can perform a comparison with the one for $N=1$ which was obtained by semiclassical methods [16, 22].

3. The model with the softly broken $O(2)$-symmetry reveals at the tree level a dynamical symmetry which is responsible for the nullification in the case of further restrictions on kinematics when the mass ratio is an integer so that no spatial momentum is involved in the decay $1 \rightarrow n$ [24]. The problem is whether this dynamical symmetry exists at arbitrary $m_{1}$ and $m_{2}$ even at the tree level.

We derive the set of recurrence relations between the multiparticle amplitudes in the large- $N$ limit at fixed number of produced particles. We transform it into a quantum mechanical problem and show how it can be obtained directly from the operator equations of motion by applying the factorization at large $N$. We find that the quantum mechanical problem can be exactly solved through the use of the Gelfand-Dikil techniques. Thus we get the generating functions for the amplitudes where the effect of loops is reduced to a renormalization of the coupling constants and masses. An interesting new phenomenon that shows up in the $O\left(N_{1}\right) \times O\left(N_{2}\right)$ model is an appearance of an imaginary part in the amplitudes which is due to inelastic processes. An extension of that result to the case of $O\left(N_{1}\right) \times \ldots \times O\left(N_{s}\right), s>2$, is shown to be straightforward. For the model of the $O(N)+$ singlet scalar particles, the generating function for the amplitudes in the large $N$ limit (no loops of a singlet field should be taken into account) can be extracted from the explicit solution for the $O\left(N_{1}\right) \times O\left(N_{2}\right)$ model.

An interesting modification of the result appears when we consider a model with a negative mass square of the singlet particle. This model corresponds to a spontaneous breaking of the parity reflection of the singlet field. The amplitudes turn out to be real for this model. By using the exact solution for this problem we demonstrate a nullification of amplitudes except for the case of production of $2 O(N)$ particles and 1 physical singlet particle.

This paper is organized as follows. Sect. 2 is devoted to the definitions and the description of the kinematics. In Sect. 3 we derive the set of recurrence relations for the $O\left(N_{1}\right) \times O\left(N_{2}\right)$ model and transform it into a quantum mechanical problem. In Sect. 4 we present an exact solution and demonstrate that it satisfies the set of equations. The exact solution is possible since the diagonal resolvent of the Schrödinger operator has a very simple form. In Sect. 5 we consider the $O(N)+$ singlet particle model with spontaneously broken parity reflection. We conclude by summarizing the results of this paper and give a generalization of the dynamical symmetry of Ref. [23] to the $O\left(N_{1}\right) \times O\left(N_{2}\right)$ type models. 


\section{Definition of the amplitudes}

We consider a model of an $O\left(N_{1}\right)$ scalar field multiplet $\chi^{a}, a=1, \ldots, N_{1}$, coupled to a $O\left(N_{2}\right)$ scalar field $\phi^{\alpha}, \alpha=1, \ldots, N_{2}$. The theory is defined in the 4-dimensional Minkowski space by the following Lagrangian

$$
\mathcal{L}=\frac{1}{2}\left(\partial_{\mu} \chi^{b}\right)\left(\partial_{\mu} \chi^{b}\right)+\frac{1}{2}\left(\partial_{\mu} \phi^{\alpha}\right)\left(\partial_{\mu} \phi^{\alpha}\right)-\frac{1}{2} m_{1}^{2}\left(\chi^{b} \chi^{b}\right)-\frac{1}{2} m_{2}^{2}\left(\phi^{\alpha} \phi^{\alpha}\right)-\frac{1}{4} \lambda\left(\phi^{\alpha} \phi^{\alpha}+\chi^{b} \chi^{b}\right)^{2}
$$

where the summation over repeated indices is implied. The interaction term is $O\left(N_{1}+N_{2}\right)$ invariant while the mass term possesses the $O\left(N_{1}\right) \times O\left(N_{2}\right)$ symmetry for nonequal masses $m_{1}$ and $m_{2}$.

We denote by $A_{b_{1} \ldots b_{n}, \alpha_{1} \ldots \alpha_{k}}^{b}(n, k)$ the amplitude of production of $n$ on-mass-shell particles $\chi$ with the $O\left(N_{1}\right)$-indices $b_{1} \ldots b_{n}$ and of $k$ on-mass-shell particles $\phi$ with the $O\left(N_{2}\right)$ indices $\alpha_{1}, \ldots, \alpha_{k}$ at rest by a (virtual) particle $\chi$ with the $O\left(N_{1}\right)$-index $b$ and the energy $n m_{1}+k m_{2}$ :

$$
A_{b_{1} \ldots b_{n}, \alpha_{1} \ldots \alpha_{k}}^{b}(n, k)=\left(\left(n m_{1}+k m_{2}\right)^{2}-m_{1}^{2}\right) m_{1}^{-n} m_{2}^{-k}\left\langle b_{1} \ldots b_{n}, \alpha_{1} \ldots \alpha_{k}\left|\chi^{b}(0)\right| 0\right\rangle .
$$

We also denote by $B_{b_{1} \ldots b_{n}, \alpha_{1} \ldots \alpha_{k}}^{\beta}(n, k)$ the amplitude of production of $n$ on-mass-shell particles $\chi$ with the $O\left(N_{1}\right)$-indices $b_{1} \ldots b_{n}$ and of $k$ on-mass-shell particles $\phi$ with $O\left(N_{2}\right)$ indices $\alpha_{1}, \ldots, \alpha_{k}$ at rest by a (virtual) particle $\phi$ with the $O\left(N_{2}\right)$-index $\beta$ and the energy $n m_{1}+k m_{2}$ :

$$
B_{b_{1} \ldots b_{n}, \alpha_{1} \ldots \alpha_{k}}^{\beta}(n, k)=\left(\left(n m_{1}+k m_{2}\right)^{2}-m_{2}^{2}\right) m_{1}^{-n} m_{2}^{-k}\left\langle b_{1} \ldots b_{n}, \alpha_{1} \ldots \alpha_{k}\left|\phi^{\beta}(0)\right| 0\right\rangle .
$$

The extra factors in Eqs. (2.2) and (2.3) are due to the amputation of the external lines.

It is convenient to multiply these amplitudes by the vectors $\xi_{1}^{b_{i}}$ and $\xi_{2}^{\alpha_{j}}$ which results in a symmetrization over the $O\left(N_{1}\right)$ and $O\left(N_{2}\right)$-indices of the produced particles and define

$$
A^{b}(n, k)=A_{b_{1} \ldots b_{n}, \alpha_{1} \ldots \alpha_{k}}^{b}(n, k) \xi_{1}^{b_{1}} \cdots \xi_{1}^{b_{n}} \xi_{2}^{\alpha_{1}} \cdots \xi_{2}^{\alpha_{k}}
$$

and

$$
B^{\alpha}(n, k)=B_{b_{1} \ldots b_{n}, \alpha_{1} \ldots \alpha_{k}}^{\alpha}(n, k) \xi_{1}^{b_{1}} \cdots \xi_{1}^{b_{n}} \xi_{2}^{\alpha_{1}} \cdots \xi_{2}^{\alpha_{k}} .
$$

The amplitudes $A^{b}(n, k)$ and $B^{\alpha}(n, k)$ are depicted graphically in Fig. 凹 $a, b$.

The other quantities which appear in the set of the recurrence relations between the amplitudes are the amplitudes $D_{b_{1} \ldots b_{n}, \alpha_{1} \ldots \alpha_{k}}^{a b}(n, k ; p)$ and $D_{b_{1} \ldots b_{n}, \alpha_{1} \ldots \alpha_{k}}^{\alpha \beta}(n, k ; p)$ of the processes when two particles $a$ and $b$ (or $\alpha$ and $\beta$ ) with the 4-momenta $p+n q_{1}+k q_{2}$ and $-p$, respectively, produce $n$ on-mass-shell particles $\chi$ with the $O\left(N_{1}\right)$-indices $b_{1}, \ldots, b_{n}$ and the equal 4-momenta $q_{1}=\left(m_{1}, 0\right)$ and $k$ on-mass-shell particles $\phi$ with the $O\left(N_{2}\right)$-indices $\alpha_{1}, \ldots, \alpha_{k}$ and the equal 4 -momenta $q_{2}=\left(m_{2}, 0\right)$ in the rest frame. We define again

$$
D_{\chi}^{a b}(n, k ; p)=D_{b_{1} \ldots b_{n}, \alpha_{1} \ldots \alpha_{k}}^{a b}(n, k ; p) \xi_{1}^{b_{1}} \cdots \xi_{1}^{b_{n}} \xi_{2}^{\alpha_{1}} \cdots \xi_{2}^{\alpha_{k}}
$$




$$
A^{a}(n, k)=\frac{n, k}{a} \quad B^{\alpha}(n, k)=-\frac{b}{b}-n, k
$$
a)
b)

Figure 1: The graphic representations of the multiparticle amplitudes $\left.A^{a}(n, k)(\mathrm{a})\right)$ and $\left.B^{\alpha}(n, k)(\mathrm{b})\right)$.

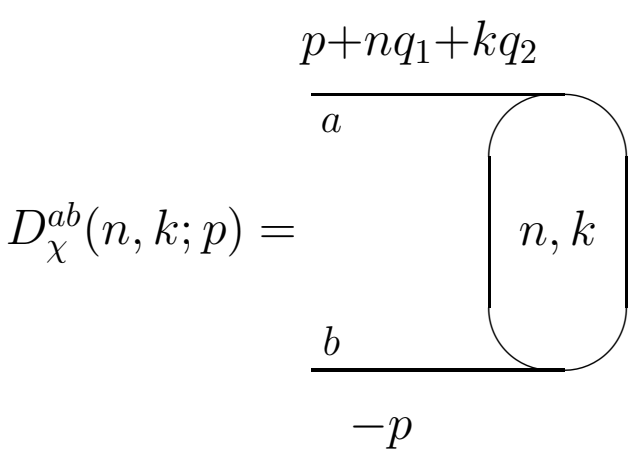

a)

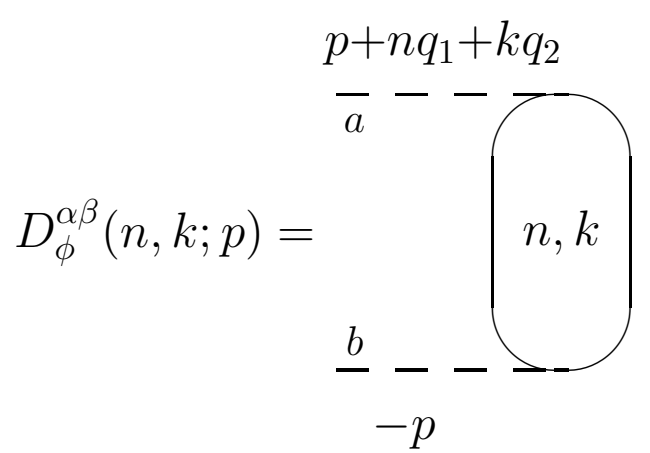

b)

Figure 2: The graphic representations of the multiparticle amplitudes $D_{\chi}^{a b}(n, k ; p)$ (a)) and $\left.D_{\phi}^{\alpha \beta}(n, k ; p)(\mathrm{b})\right)$. 
and

$$
D_{\phi}^{\alpha \beta}(n, k ; p)=D_{b_{1} \ldots b_{n}, \alpha_{1} \ldots \alpha_{k}}^{\alpha \beta}(n, k ; p) \xi_{1}^{b_{1}} \cdots \xi_{1}^{b_{n}} \xi_{2}^{\alpha_{1}} \cdots \xi_{2}^{\alpha_{k}} .
$$

The amplitudes $D_{\chi}^{a b}(n, k ; p)$ and $D_{\phi}^{\alpha \beta}(n, k ; p)$ are depicted graphically in Fig. 2 $a, b$. The definitions (2.4), (2.5), 2.6) and (2.7) generalize those of Ref. [26] to the case of nonequal masses $m_{1}$ and $m_{2}$.

Notice that we do not introduce the amplitudes associated with the mixed case when the incoming particles are $\chi$ and $\phi$. This is because the unsuppressed in the large $N$ limit loop diagrams contain only loops of $\chi_{b}$ and $\phi_{\alpha}$ particles with contracted $O\left(N_{1,2}\right)$ indices (it is assumed that $\lambda \sim 1 / N)$. Notice also that in the case $N_{1}=N \rightarrow \infty$ and $N_{2}=$ finite we can simply drop out the contributions from the loops with $\phi$ virtual particles. That means that in this case we have to consider only $D_{\chi}^{a b}$ vertices. Actually we shall see that the amplitudes for the $N_{1}=N \rightarrow \infty, N_{2}=$ finite case can be easily extracted from the expression for the amplitudes given below for the case $N_{1,2} \rightarrow \infty$ by taking formally $N_{2} / N_{1}=0$.

It is easy to estimate at the tree level that

$$
A^{b}(n, k) \sim \lambda^{\frac{n+k-1}{2}}\left(\xi_{1}^{2}\right)^{\frac{n-1}{2}}\left(\xi_{2}^{2}\right)^{\frac{k-1}{2}} \xi_{1}^{b}, \quad B^{\beta}(n, k) \sim \lambda^{\frac{n+k-1}{2}}\left(\xi_{1}^{2}\right)^{\frac{n-1}{2}}\left(\xi_{2}^{2}\right)^{\frac{k-1}{2}} \xi_{2}^{\beta},
$$

where $\xi_{1}^{2}$ and $\xi_{2}^{2}$ stand for $\xi_{1}^{a} \xi_{1}^{a}$ and $\xi_{2}^{\alpha} \xi_{2}^{\alpha}$ respectively. Since

$$
\lambda \sim \frac{1}{N}
$$

in the large- $N$ limit 28], we choose

$$
\xi_{1}^{2} \sim \xi_{2}^{2} \sim N
$$

for all the amplitudes (2.8) to be of the same order in $1 / N$.

\section{Schwinger-Dyson equations at large $N$}

\subsection{The diagrammatic derivation}

The condition (2.9) leaves at large- $N$ only the diagrams with the largest possible number of sums over internal $O\left(N_{1,2}\right)$-indices which propagate along closed loops of diagrams. The proper recurrence relations which extend the usual Schwinger-Dyson equations to the case when $n$ particles $\chi$ and $k$ particles $\phi$ are produced are depicted for $A^{b}(n, k)$ and $B^{\alpha}(n, k)$ in Fig. 3. The solid lines are associated with the propagation of the $O\left(N_{1}\right)$ indices (the field $\chi$ ), while the dashed ones stand for the propagation of the $O\left(N_{2}\right)$ indices (the field $\phi$ ). Each vertex is the sum of three possible permutations of the $O(N)$-indices. This is taken into account in Fig. 3 by a combinatorial factor. The graphic notations becomes clear if one introduces the auxiliary field $\sigma(x)=\chi^{2}(x)+\phi^{2}(x)$ which propagates in the empty space inside vertices. 

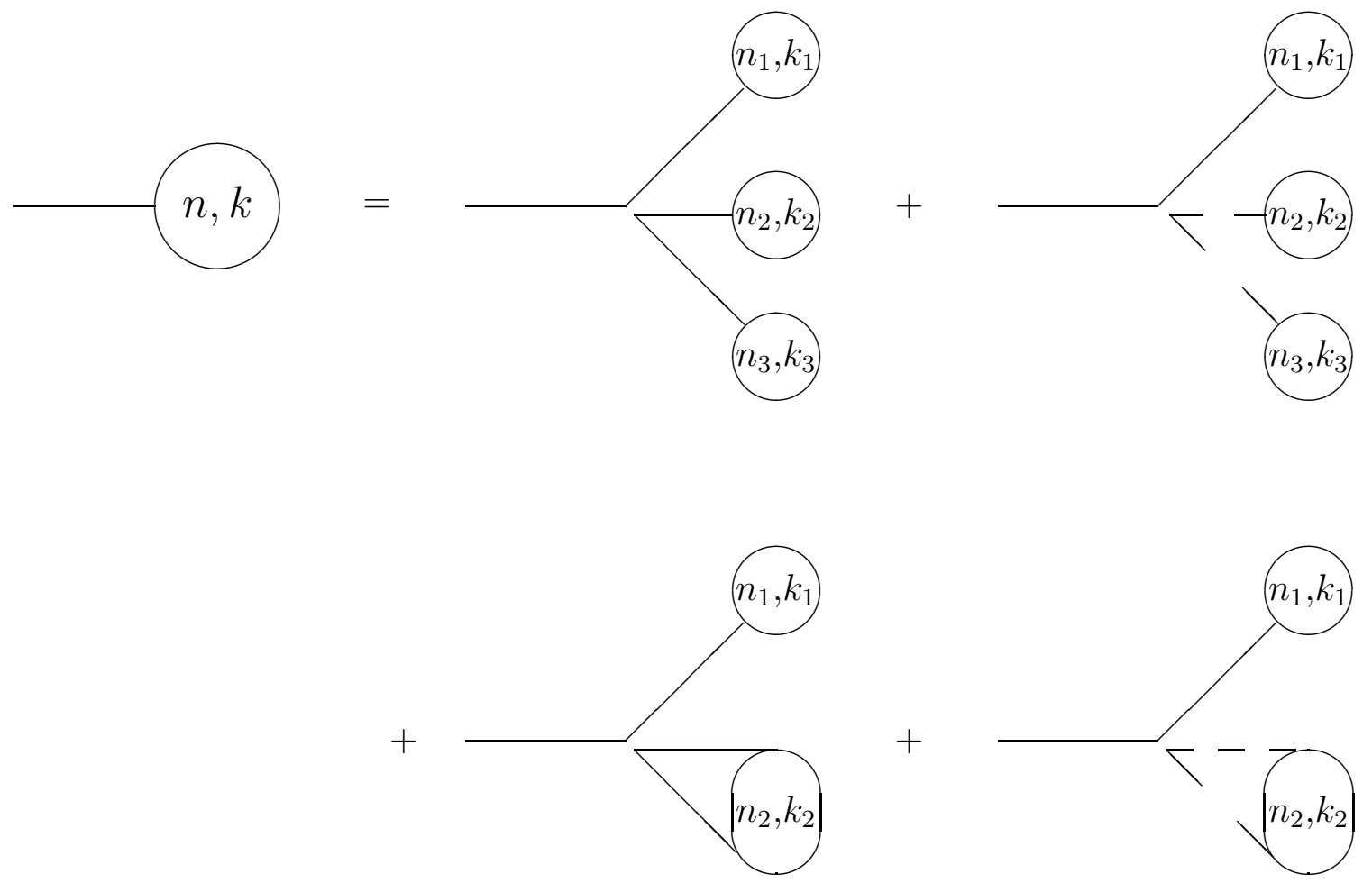

Figure 3: The recurrence relation for the amplitude $A^{a}(n, k)$. The analytical formula is given by Eq. (3.1). The analogous recurrence relation for the amplitude $B^{\alpha}(n, k)$ can be obtained by interchanging the solid and dashed lines. 
The recurrence relations of Fig. 3 read analytically as follows

$$
\begin{gathered}
A^{a}(n, k)=\lambda \sum_{\substack{n_{i}=\text { odd, } \\
k_{i}=\text { even }}} \delta_{n, n_{1}+n_{2}+n_{3}} \delta_{k, k_{1}+k_{2}+k_{3}} \frac{n ! k !}{n_{1} ! n_{2} ! n_{3} ! k_{1} ! k_{2} ! k_{3} !} \\
\frac{A^{a}\left(n_{1}, k_{1}\right) A^{b}\left(n_{2}, k_{2}\right) A^{b}\left(n_{3}, k_{3}\right)}{\left(\left(n_{1} m_{1}+k_{1} m_{2}\right)^{2}-m_{1}^{2}\right)\left(\left(n_{2} m_{1}+k_{2} m_{2}\right)^{2}-m_{1}^{2}\right)\left(\left(n_{3} m_{1}+k_{3} m_{2}\right)^{2}-m_{1}^{2}\right)}+ \\
+\lambda \sum_{\substack{n_{1}, k_{2}, k_{3}=\text { odd, } \\
n_{2}, n_{3}, k_{1}=e v e n}} \delta_{n, n_{1}+n_{2}+n_{3}} \delta_{k, k_{1}+k_{2}+k_{3}} \frac{n ! k !}{n_{1} ! n_{2} ! n_{3} ! k_{1} ! k_{2} ! k_{3} !} \\
\frac{A^{a}\left(n_{1}, k_{1}\right) B^{\alpha}\left(n_{2}, k_{2}\right) B^{\alpha}\left(n_{3}, k_{3}\right)}{\left(\left(n_{1} m_{1}+k_{1} m_{2}\right)^{2}-m_{1}^{2}\right)\left(\left(n_{2} m_{1}+k_{2} m_{2}\right)^{2}-m_{2}^{2}\right)\left(\left(n_{3} m_{1}+k_{3} m_{2}\right)^{2}-m_{2}^{2}\right)}+ \\
+\sum_{\substack{n_{1}=\text { odd, } \\
n_{2}, k_{1}, k_{2}=\text { even }}} \delta_{n, n_{1}+n_{2}} \delta_{k, k_{1}+k_{2}} \frac{n ! k !}{n_{1} ! n_{2} ! k_{1} ! k_{2} !} \\
\frac{A^{a}\left(n_{1}, k_{1}\right)}{\left(\left(n_{1} m_{1}+k_{1} m_{2}\right)^{2}-m_{1}^{2}\right)} \int \frac{d^{4} p}{(2 \pi)^{4}}\left(D_{\chi}^{b b}\left(n_{2}, k_{2} ; p\right)+D_{\phi}^{\alpha \alpha}\left(n_{2}, k_{2} ; p\right)\right)
\end{gathered}
$$

and similarly

$$
\begin{gathered}
B^{\alpha}(n, k)=\lambda \sum_{\substack{k_{1}, n_{2}, n_{3}=\text { odd, } \\
n_{1}, k_{2}, k_{3}=\text { even }}} \delta_{n, n_{1}+n_{2}+n_{3}} \delta_{k, k_{1}+k_{2}+k_{3}} \frac{n ! k !}{n_{1} ! n_{2} ! n_{3} ! k_{1} ! k_{2} ! k_{3} !} \\
\frac{B^{\alpha}\left(n_{1}, k_{1}\right) A^{b}\left(n_{2}, k_{2}\right) A^{b}\left(n_{3}, k_{3}\right)}{\left(\left(n_{1} m_{1}+k_{1} m_{2}\right)^{2}-m_{2}^{2}\right)\left(\left(n_{2} m_{1}+k_{2} m_{2}\right)^{2}-m_{1}^{2}\right)\left(\left(n_{3} m_{1}+k_{3} m_{2}\right)^{2}-m_{1}^{2}\right)}+ \\
+\lambda \sum_{\substack{k_{i}=\text { odd, } \\
n_{i}=e v e n}} \delta_{n, n_{1}+n_{2}+n_{3}} \delta_{k, k_{1}+k_{2}+k_{3}} \frac{n ! k !}{n_{1} ! n_{2} ! n_{3} ! k_{1} ! k_{2} ! k_{3} !} \\
\frac{B^{\alpha}\left(n_{1}, k_{1}\right) B^{\beta}\left(n_{2}, k_{2}\right) B^{\beta}\left(n_{3}, k_{3}\right)}{\left(\left(n_{1} m_{1}+k_{1} m_{2}\right)^{2}-m_{2}^{2}\right)\left(\left(n_{2} m_{1}+k_{2} m_{2}\right)^{2}-m_{2}^{2}\right)\left(\left(n_{3} m_{1}+k_{3} m_{2}\right)^{2}-m_{2}^{2}\right)}+ \\
+\lambda \sum_{\substack{k_{1}=\text { odd, } \\
n_{1}, n_{2}, k_{2}=\text { even }}} \delta_{n, n_{1}+n_{2}} \delta_{k, k_{1}+k_{2}} \frac{n ! k !}{n_{1} ! n_{2} ! k_{1} ! k_{2} !} \\
\frac{B^{\alpha}\left(n_{1}, k_{1}\right)}{\left(\left(n_{1} m_{1}+k_{1} m_{2}\right)^{2}-m_{2}^{2}\right)} \int \frac{d^{4} p}{(2 \pi)^{4}}\left(D_{\chi}^{b b}\left(n_{2}, k_{2} ; p\right)+D_{\phi}^{\alpha \alpha}\left(n_{2}, k_{2} ; p\right)\right) .
\end{gathered}
$$

Notice that only the summed over the $O\left(N_{1}\right)$ and $O\left(N_{2}\right)$-indices quantities $D_{\chi}^{b b}\left(n_{2}, k_{2} ; p\right)$ and $D_{\phi}^{\alpha \alpha}\left(n_{2}, k_{2} ; p\right)$ enter these recurrence relations.

It is the property of large $N_{1,2}$ that the recurrence relations for $D_{\chi}^{a a}(n, k ; p)$ and $D_{\chi}^{a a}(n, k ; p)$ express it via $A^{b}, B^{\alpha}$ and $D_{\chi}^{b b}, D_{\phi}^{\alpha \alpha}$ again. The recurrence relations for $D_{\chi}^{a a}(n, k ; p)$ and $D_{\phi}^{\alpha \alpha}(n, k ; p)$ are depicted in Fig. 4 . 

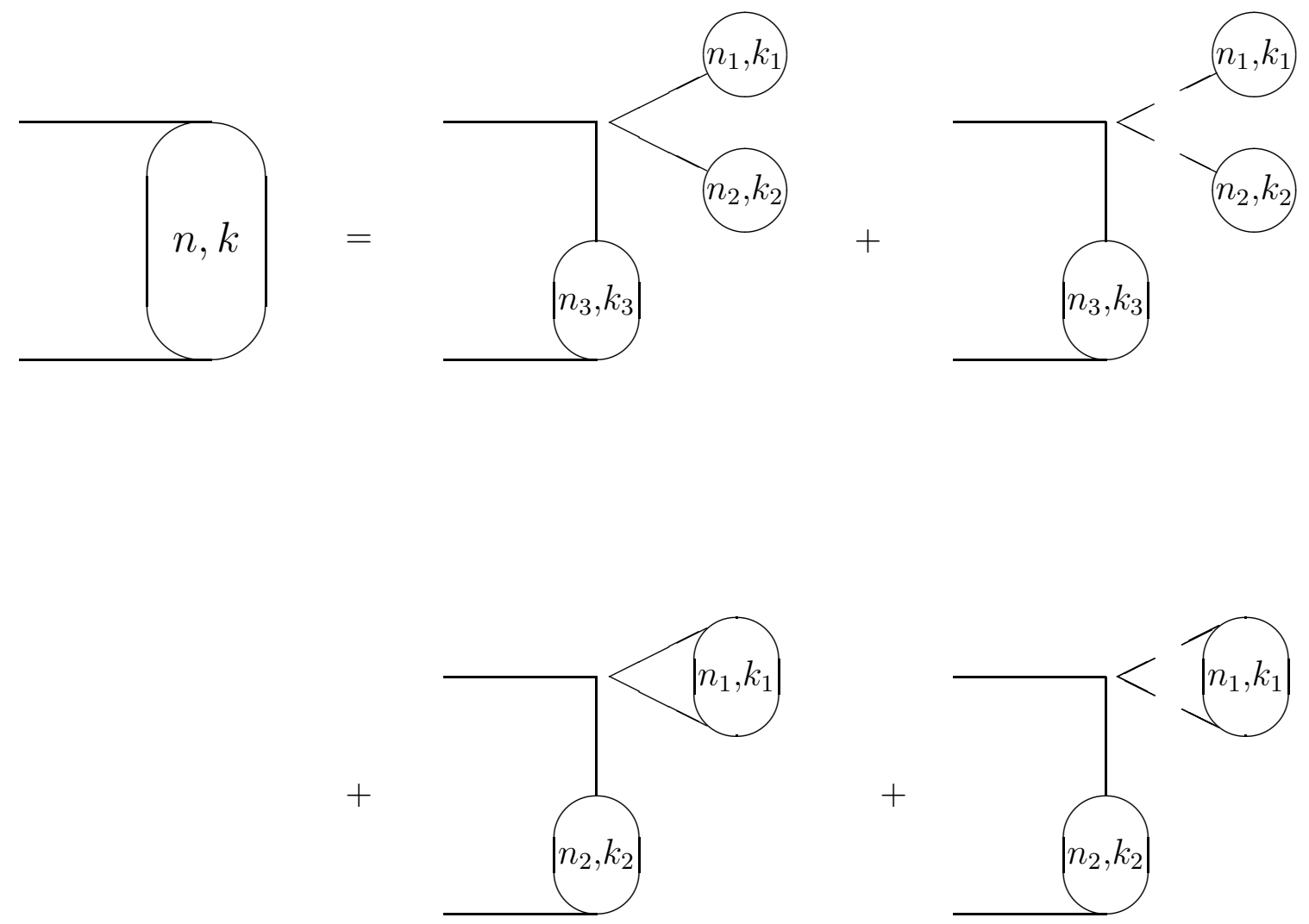

Figure 4: The recurrence relation for the amplitude $D_{\chi}^{a a}(n, k ; p)$. The analytical formula is given by Eq. (3.3). The recurrence relation for the amplitude $D_{\phi}^{\alpha \alpha}(n, k ; p)$ can be obtained by interchanging the solid and dashed lines. 
The recurrence relation of Fig. 14 reads analytically as follows

$$
\begin{array}{r}
D_{\chi}^{a a}(n, k)=\lambda \sum_{\substack{n_{1}, n_{2}=\text { odd, } \\
n_{3}, k_{i}=\text { even }}} \delta_{n, n_{1}+n_{2}+n_{3}} \delta_{k, k_{1}+k_{2}+k_{3}} \frac{n ! k !}{n_{1} ! n_{2} ! n_{3} ! k_{1} ! k_{2} ! k_{3} !} \\
\frac{1}{\left(p+n_{3} q_{1}+k_{3} q_{2}\right)^{2}-m_{1}^{2}} \frac{A^{b}\left(n_{1}, k_{1}\right) A^{b}\left(n_{2}, k_{2}\right) D_{\chi}^{a a}\left(n_{3}, k_{3} ; p\right)}{\left(\left(n_{1} m_{1}+k_{1} m_{2}\right)^{2}-m_{1}^{2}\right)\left(\left(n_{2} m_{1}+k_{2} m_{2}\right)^{2}-m_{1}^{2}\right)}+ \\
+\lambda \sum_{\substack{k_{1}, k_{2}=\text { odd, } \\
n_{i}, k_{3}=\text { even }}} \delta_{n, n_{1}+n_{2}+n_{3}} \delta_{k, k_{1}+k_{2}+k_{3}} \frac{n ! k !}{n_{1} ! n_{2} ! n_{3} ! k_{1} ! k_{2} ! k_{3} !} \\
\frac{B^{\beta}\left(n_{1}, k_{1}\right) B^{\beta}\left(n_{2}, k_{2}\right) D_{\chi}^{a a}\left(n_{3}, k_{3} ; p\right)}{\left(p+n_{3} q_{1}+k_{3} q_{2}\right)^{2}-m_{1}^{2}} \frac{\sum_{\left.\left(n_{1} m_{1}+k_{1} m_{2}\right)^{2}-m_{2}^{2}\right)\left(\left(n_{2} m_{1}+k_{2} m_{2}\right)^{2}-m_{2}^{2}\right)}+}{\quad+\lambda \sum_{n_{i}, k_{i}=\text { even }} \delta_{n, n_{1}+n_{2}} \delta_{k, k_{1}+k_{2}} \frac{n ! k !}{n_{1} ! n_{2} ! k_{1} ! k_{2} !}} \\
\left.\frac{d^{4} k}{\left(p+n_{2} q_{1}+k_{2} q_{2}\right)^{2}-m_{1}^{2}} \int D_{\chi}^{b b}\left(n_{1}, k_{1} ; k\right)+D_{\phi}^{\beta \beta}\left(n_{1}, k_{1} ; k\right)\right) D_{\chi}^{a a}\left(n_{2}, k_{2} ; p\right) .
\end{array}
$$

Similarly we have

$$
\begin{aligned}
& D_{\phi}^{\alpha \alpha}(n, k)= \lambda \sum_{\substack{n_{1}, n_{2}=\text { odd, } \\
n_{3}, k_{i}=\text { even }}} \delta_{n, n_{1}+n_{2}+n_{3}} \delta_{k, k_{1}+k_{2}+k_{3}} \frac{n ! k !}{n_{1} ! n_{2} ! n_{3} ! k_{1} ! k_{2} ! k_{3} !} \\
& \frac{1}{\left(p+n_{3} q_{1}+k_{3} q_{2}\right)^{2}-m_{2}^{2}} \frac{A^{b}\left(n_{1}, k_{1}\right) A^{b}\left(n_{2}, k_{2}\right) D_{\phi}^{\alpha \alpha}\left(n_{3}, k_{3} ; p\right)}{\left(\left(n_{1} m_{1}+k_{1} m_{2}\right)^{2}-m_{1}^{2}\right)\left(\left(n_{2} m_{1}+k_{2} m_{2}\right)^{2}-m_{1}^{2}\right)}+ \\
&+\lambda \sum_{\substack{k_{1}, k_{2}=\text { odd, } \\
k_{3}, n_{i}=\text { even }}} \delta_{n, n_{1}+n_{2}+n_{3}} \delta_{k, k_{1}+k_{2}+k_{3}} \frac{n ! k !}{n_{1} ! n_{2} ! n_{3} ! k_{1} ! k_{2} ! k_{3} !} \\
& \frac{B^{\beta}\left(n_{1}, k_{1}\right) B^{\beta}\left(n_{2}, k_{2}\right) D_{\phi}^{\alpha \alpha}\left(n_{3}, k_{3} ; p\right)}{\left(p+n_{3} q_{1}+k_{3} q_{2}\right)^{2}-m_{2}^{2}} \frac{\sum_{\left.\left(n_{1} m_{1}+k_{1} m_{2}\right)^{2}-m_{2}^{2}\right)\left(\left(n_{2} m_{1}+k_{2} m_{2}\right)^{2}-m_{2}^{2}\right)}+}{+\sum_{n_{i}, k_{i}=e v e n} \delta_{n, n_{1}+n_{2}} \delta_{k, k_{1}+k_{2}} \frac{n ! k !}{n_{1} ! n_{2} ! k_{1} ! k_{2} !}} \\
& \frac{d^{4} k}{(2 \pi)^{4}}\left(D_{\chi}^{b b}\left(n_{1}, k_{1} ; k\right)+D_{\phi}^{\beta \beta}\left(n_{1}, k_{1} ; k\right)\right) D_{\phi}^{\alpha \alpha}\left(n_{2}, k_{2} ; p\right) .
\end{aligned}
$$

Eqs. (3.1), (3.2), (3.3) and (3.4) look very similar to the ones [26 for the $O(N)$-case.

To rewrite Eqs. (3.1), (3.2), (3.3) and (3.4) in a more convenient form, let us introduce the generating functions

$$
\begin{aligned}
& \Phi^{a}(\tau)=m_{1} \xi_{1}^{a} \mathrm{e}^{m_{1} \tau}+\sum_{n, k \geq 3} \frac{A^{a}(n, k)}{n ! k !\left(\left(n m_{1}+k m_{2}\right)^{2}-m_{1}^{2}\right)} \mathrm{e}^{\left(n m_{1}+k m_{2}\right) \tau} m_{1}^{n} m_{2}^{k}, \\
& \Psi^{\alpha}(\tau)=m_{2} \xi_{2}^{\alpha} \mathrm{e}^{m_{2} \tau}+\sum_{n, k \geq 3} \frac{B^{\alpha}(n, k)}{n ! k !\left(\left(n m_{1}+k m_{2}\right)^{2}-m_{2}^{2}\right)} \mathrm{e}^{\left(n m_{1}+k m_{2}\right) \tau} m_{2}^{n} m_{2}^{k},
\end{aligned}
$$

\footnotetext{
${ }^{4}$ Here and below the poles should be understood according to the Feynman prescription $m^{2} \rightarrow m^{2}-i 0$.
} 


$$
D_{\chi}^{a b}(\tau ; p)=\frac{i \delta^{a b}}{p^{2}-m_{1}^{2}}+\sum_{n, k=\text { even }} D_{\chi}^{a b}(n, k ; p) \frac{1}{n ! k !} \mathrm{e}^{\left(n m_{1}+k m_{2}\right) \tau} m_{1}^{n} m_{2}^{k}
$$

and

$$
D_{\phi}^{\alpha \beta}(\tau ; p)=\frac{i \delta^{\alpha \beta}}{p^{2}-m_{2}^{2}}+\sum_{n, k=\text { even }} D_{\phi}^{\alpha \beta}(n, k ; p) \frac{1}{n ! k !} \mathrm{e}^{\left(n m_{1}+k m_{2}\right) \tau} m_{1}^{n} m_{2}^{k} .
$$

Eqs. (3.1), (3.2), (3.3) and (3.4) can then be rewritten, respectively, as

$$
\begin{aligned}
& \left\{\frac{d^{2}}{d \tau^{2}}-m_{1}^{2}-v(\tau)\right\} \Phi^{a}(\tau)=0 \\
& \left\{\frac{d^{2}}{d \tau^{2}}-m_{2}^{2}-v(\tau)\right\} \Psi^{\alpha}(\tau)=0
\end{aligned}
$$

and

$$
\begin{gathered}
\left\{\frac{d^{2}}{d \tau^{2}}-\omega_{1}^{2}-v(\tau)\right\} \mathrm{e}^{\epsilon \tau} \frac{1}{N_{1}} D_{\chi}^{b b}(\tau ; p)=\mathrm{e}^{\epsilon \tau}, \\
\left\{\frac{d^{2}}{d \tau^{2}}-\omega_{2}^{2}-v(\tau)\right\} \mathrm{e}^{\epsilon \tau} \frac{1}{N_{2}} D_{\phi}^{b b}(\tau ; p)=\mathrm{e}^{\epsilon \tau}
\end{gathered}
$$

where $\epsilon$ is the energy component of $p, p=(\epsilon, \vec{p})$,

$$
\omega_{1,2}=\sqrt{\vec{p}^{2}+m_{1,2}^{2}}
$$

and

$$
v(\tau)=\lambda\left(\Phi^{2}(\tau)+\Psi^{2}(\tau)\right)+\lambda \int \frac{d^{4} p}{(2 \pi)^{4}}\left(D_{\chi}^{b b}(\tau ; p)+D_{\phi}^{\beta \beta}(\tau ; p)\right) .
$$

The next step in the transformation of Eqs. (3.9), (3.10), (3.11), (3.12) and (3.14) is to put $D_{\chi}^{a b}$ and $D_{\phi}^{\alpha \beta}$ in the mixed representation - the coordinate in (imaginary) time and momentum for space. One defines

$$
\begin{aligned}
& D_{\chi}^{a b}\left(\omega_{1} ; \tau, \tau^{\prime}\right)=\int \frac{d \epsilon}{2 \pi} \mathrm{e}^{\epsilon\left(\tau-\tau^{\prime}\right)} D_{\chi}^{a b}(\tau ; p), \\
& D_{\phi}^{\alpha \beta}\left(\omega_{2} ; \tau, \tau^{\prime}\right)=\int \frac{d \epsilon}{2 \pi} \mathrm{e}^{\epsilon\left(\tau-\tau^{\prime}\right)} D_{\phi}^{\alpha \beta}(\tau ; p) .
\end{aligned}
$$

In order to see that this quantity is indeed associated with the Fourier transform of amplitudes in energy, we notice that the insertion of Eq. (3.7) on the r.h.s. of Eq. (3.15) yields

$$
D_{\chi}^{a b}\left(\omega_{1} ; \tau, \tau^{\prime}\right)=\int \frac{d \epsilon}{2 \pi} \sum_{n=0}^{\infty} \mathrm{e}^{\left(\epsilon+n m_{1}+k m_{2}\right) \tau-\epsilon \tau^{\prime}} D_{\chi}^{a b}(n ; p) .
$$

One recognizes now that $\epsilon+n m_{1}+k m_{2}$ is the energy component of the 4-momentum $p+n q_{1}+k q_{2}$ of the incoming particle with the $O\left(N_{1}\right)$-index $a$ while $\epsilon$ is that of $p$ for $b$. In particular, the free propagator in the mixed representation is given by

$$
D_{\chi}^{a b}\left(\omega_{1} ; \tau, \tau^{\prime}\right)=i \delta^{a b} \int \frac{d \epsilon}{2 \pi} \frac{\mathrm{e}^{\epsilon\left(\tau-\tau^{\prime}\right)}}{\left(\epsilon^{2}-\omega_{1}^{2}+i 0\right)}=\delta^{a b} \frac{1}{2 \omega_{1}} \mathrm{e}^{-\omega_{1}\left|\tau-\tau^{\prime}\right|} \quad(\text { for } \lambda=0) .
$$


The equations (3.11), (3.12) and (3.14) can be finally rewritten in the mixed representation as follows:

$$
\begin{aligned}
& \left\{\frac{d^{2}}{d \tau^{2}}-\omega^{2}-v(\tau)\right\} \frac{1}{N_{1}} D_{\chi}^{b b}\left(\omega ; \tau, \tau^{\prime}\right)=-\delta\left(\tau-\tau^{\prime}\right), \\
& \left\{\frac{d^{2}}{d \tau^{2}}-\omega^{2}-v(\tau)\right\} \frac{1}{N_{2}} D_{\phi}^{\beta \beta}\left(\omega ; \tau, \tau^{\prime}\right)=-\delta\left(\tau-\tau^{\prime}\right)
\end{aligned}
$$

and

$$
\begin{gathered}
v(\tau)=\lambda\left(\Phi^{2}(\tau)+\Psi^{2}(\tau)\right)+\frac{\lambda}{2 \pi^{2}}\left(\int_{m_{1}} d \omega \omega \sqrt{\omega^{2}-m_{1}^{2}} D_{\chi}^{b b}(\omega ; \tau, \tau)+\right. \\
\left.+\int_{m_{2}} d \omega \omega \sqrt{\omega^{2}-m_{2}^{2}} D_{\phi}^{\beta \beta}(\omega ; \tau, \tau)\right) .
\end{gathered}
$$

To understand the meaning of the summed amplitudes $D_{\chi}^{b b}\left(\omega ; \tau, \tau^{\prime}\right)$ and $D_{\phi}^{\beta \beta}\left(\omega ; \tau, \tau^{\prime}\right)$, let us note that $D_{\chi}^{b c}\left(\omega ; \tau, \tau^{\prime}\right)$ has in the index space the structure

$$
D_{\chi}^{b c}\left(\omega ; \tau, \tau^{\prime}\right)=\left(\delta^{b c}-\frac{\xi_{1}^{b} \xi_{1}^{c}}{\xi_{1}^{2}}\right) G_{\chi}^{T}\left(\omega ; \tau, \tau^{\prime}\right)+\frac{\xi_{1}^{b} \xi_{1}^{c}}{\xi_{1}^{2}} G_{\chi}^{S}\left(\omega ; \tau, \tau^{\prime}\right)
$$

The amplitudes $G^{T}$ and $G^{S}$ are associated, respectively, with the tensor and singlet $O\left(N_{1}\right)$-states of the two incoming particles. The averaged over the $O\left(N_{1}\right)$-indices of two incoming particles amplitude is

$$
\frac{1}{N_{1}} D_{\chi}^{b b}\left(\omega ; \tau, \tau^{\prime}\right)=\left(1-\frac{1}{N_{1}}\right) G_{\chi}^{T}\left(\omega ; \tau, \tau^{\prime}\right)+\frac{1}{N_{1}} G_{\chi}^{S}\left(\omega ; \tau, \tau^{\prime}\right)
$$

while the generating function for the symmetrized over all the $n+2 O\left(N_{1}\right)$-indices amplitudes is given by $G^{S}$ :

$$
D_{\chi}^{a b}\left(\omega ; \tau, \tau^{\prime}\right) \frac{\xi_{1}^{a} \xi_{1}^{b}}{\xi_{1}^{2}}=G_{\chi}^{S}\left(\omega ; \tau, \tau^{\prime}\right)
$$

One sees that the averaged amplitude which enters Eq. (3.21) coincides with $G_{\chi}^{T}$ at large $N_{1}$.

We can now rewrite Eqs. (3.19) and (3.20) at large $N$ as the following equations for $G_{\chi}^{T}\left(\omega ; \tau, \tau^{\prime}\right)$ and $G_{\phi}^{T}\left(\omega ; \tau, \tau^{\prime}\right)$ (the latter is defined similarly to Eq. (3.23):

$$
\begin{aligned}
& \left\{\frac{d^{2}}{d \tau^{2}}-\omega^{2}-v(\tau)\right\} G_{\chi}^{T}\left(\omega ; \tau, \tau^{\prime}\right)=-\delta\left(\tau-\tau^{\prime}\right), \\
& \left\{\frac{d^{2}}{d \tau^{2}}-\omega^{2}-v(\tau)\right\} G_{\phi}^{T}\left(\omega ; \tau, \tau^{\prime}\right)=-\delta\left(\tau-\tau^{\prime}\right),
\end{aligned}
$$

while $v(\tau)$ is related to $G_{\chi}^{T}(\omega ; \tau, \tau)$ and $G_{\phi}^{T}(\omega ; \tau, \tau)$ by

$$
\begin{aligned}
v(\tau)=\lambda\left(\Phi^{2}(\tau)+\Psi^{2}(\tau)\right) & +\frac{\lambda N_{1}}{2 \pi^{2}} \int_{m_{1}} d \omega \omega \sqrt{\omega^{2}-m_{1}^{2}} G_{\chi}^{T}(\omega ; \tau, \tau)+ \\
+ & \frac{\lambda N_{2}}{2 \pi^{2}} \int_{m_{2}} d \omega \omega \sqrt{\omega^{2}-m_{2}^{2}} G_{\phi}^{T}(\omega ; \tau, \tau) .
\end{aligned}
$$


Therefore, only the tensor amplitudes enter at large $N_{1,2}$.

The transformation from Eqs. (3.1), (3.2), (3.3) and (3.4) into Eqs. (3.9), (3.10), (3.25), (3.26) and (3.27) is similar to the one of Refs. [0, 12, 15, 20, 26]. These equations form the closed set which will be solved in the next section.

\subsection{The operator derivation}

The structure of Eqs. (3.9), (3.10), (3.25), (3.26) and (3.27) is easy to understand through the use of the functional technique [10] which relates $\tau$ to the time variable $t \equiv x_{0}$ of the fields $\chi^{a}(x)$ and $\phi^{\alpha}(x)$ by

$$
\tau=i t
$$

Using the definitions (2.2) and (2.3), one rewrites (3.5) and (3.6) as

$$
\begin{aligned}
\Phi^{a}(\tau)= & m_{1} \xi_{1}^{a} \mathrm{e}^{m_{1} \tau} \\
& +\sum_{n, k \geq 3} \frac{1}{n ! k !}\left\langle b_{1} \ldots b_{n}, \beta_{1} \ldots \beta_{k}\left|\chi^{a}(0)\right| 0\right\rangle \xi_{1}^{b_{1}} \ldots \xi_{1}^{b_{n}} \xi_{2}^{\beta_{1}} \ldots \xi_{2}^{\beta_{k}} \mathrm{e}^{\left(n m_{1}+k m_{2}\right) \tau} \\
= & \sum_{n, k \geq 1} \frac{1}{n ! k !}\left\langle b_{1} \ldots b_{n}, \beta_{1} \ldots \beta_{k}\left|\chi^{a}(\overrightarrow{0},-i \tau)\right| 0\right\rangle \xi_{1}^{b_{1}} \ldots \xi_{1}^{b_{n}} \xi_{2}^{\beta_{1}} \ldots \xi_{2}^{\beta_{k}}
\end{aligned}
$$

and

$$
\begin{aligned}
\Psi^{\alpha}(\tau)= & m_{2} \xi_{2}^{\alpha} \mathrm{e}^{m_{2} \tau} \\
& +\sum_{n, k \geq 3} \frac{1}{n ! k !}\left\langle b_{1} \ldots b_{n}, \beta_{1} \ldots \beta_{k}\left|\phi^{\alpha}(0)\right| 0\right\rangle \xi_{1}^{b_{1}} \ldots \xi_{1}^{b_{n}} \xi_{2}^{\beta_{1}} \ldots \xi_{2}^{\beta_{k}} \mathrm{e}^{\left(n m_{1}+k m_{2}\right) \tau} \\
= & \sum_{n, k \geq 1} \frac{1}{n ! k !}\left\langle b_{1} \ldots b_{n}, \beta_{1} \ldots \beta_{k}\left|\phi^{\alpha}(\overrightarrow{0},-i \tau)\right| 0\right\rangle \xi_{1}^{b_{1}} \ldots \xi_{1}^{b_{n}} \xi_{2}^{\beta_{1}} \ldots \xi_{2}^{\beta_{k}} .
\end{aligned}
$$

Similarly, using Eq. (3.7) and (3.8), one sees that $v(\tau)$ which is defined by Eq. (3.14) is nothing but the sum of the matrix elements:

$$
\begin{aligned}
v(\tau)= & \\
& \lambda \sum_{n, k=0}^{\infty} \frac{1}{n ! k !}\left\langle b_{1} \ldots b_{n}, \beta_{1} \ldots \beta_{k}\left|:\left(\chi^{2}(0)+\phi^{2}(0)\right):\right| 0\right\rangle \xi_{1}^{b_{1}} \cdots \xi_{1}^{b_{n}} \xi_{2}^{\beta_{1}} \ldots \xi_{2}^{\beta_{k}} \mathrm{e}^{\left(n m_{1}+k m_{2}\right) \tau} \\
= & \lambda \sum_{n, k=0}^{\infty} \frac{1}{n ! k !}\left\langle b_{1} \ldots b_{n}, \beta_{1} \ldots \beta_{k}\left|:\left(\chi^{2}(\overrightarrow{0},-i \tau)+\phi^{2}(\overrightarrow{0},-i \tau)\right):\right| 0\right\rangle \xi_{1}^{b_{1}} \ldots \xi_{1}^{b_{n}} \xi_{2}^{\beta_{1}} \ldots \xi_{2}^{\beta_{k}}
\end{aligned}
$$

where : ... : stands for a normal odering.

To simplify the notations, let us introduce the coherent state

$$
\langle\Xi|=\sum_{n, k=0}^{\infty} \frac{1}{n ! k !} \xi_{1}^{b_{1}} \cdots \xi_{1}^{b_{n}} \xi_{2}^{\beta_{1}} \ldots \xi_{2}^{\beta_{k}}\left\langle b_{1} \ldots b_{n}, \beta_{1} \ldots \beta_{k}\right| .
$$


Then Eqs. (3.29), (3.30) and (3.31) can be written as follows:

$$
\begin{gathered}
\Phi^{a}(\tau)=\left\langle\Xi\left|\chi^{a}(\overrightarrow{0},-i \tau)\right| 0\right\rangle \\
\Psi^{\alpha}(\tau)=\left\langle\Xi\left|\phi^{\alpha}(\overrightarrow{0},-i \tau)\right| 0\right\rangle
\end{gathered}
$$

and

$$
v(\tau)=\left\langle\Xi\left|:\left(\chi^{2}(\overrightarrow{0},-i \tau)+\phi^{2}(\overrightarrow{0},-i \tau)\right):\right| 0\right\rangle .
$$

Eqs. (3.9), (3.10), (3.25), (3.26) and (3.27) can alternatively be deduced directly from the operator equations for the quantum fields $\chi^{a}$ and $\phi^{\alpha}$ :

$$
\left(-\partial_{\mu} \partial_{\mu}-m_{1}^{2}\right) \chi^{a}-\lambda:\left(\chi^{2}+\phi^{2}\right) \chi^{a}:=0
$$

and

$$
\left(-\partial_{\mu} \partial_{\mu}-m_{2}^{2}\right) \phi^{\alpha}-\lambda:\left(\chi^{2}+\phi^{2}\right) \phi^{\alpha}:=0
$$

Let us take the matrix elements of Eqs. (3.36) and (3.37) between the states $\langle\Xi|$ and $|0\rangle$. We get

$$
\begin{array}{r}
\left\{\frac{\partial^{2}}{\partial \tau^{2}}+\frac{\partial^{2}}{\partial \vec{x}^{2}}-m_{1}^{2}\right\}\left\langle\Xi\left|\chi^{a}(\vec{x},-i \tau)\right| 0\right\rangle \\
-\lambda\left\langle\Xi\left|:\left(\chi^{2}(\vec{x},-i \tau)+\phi^{2}(\vec{x},-i \tau)\right) \chi^{a}(\vec{x},-i \tau):\right| 0\right\rangle=0,
\end{array}
$$

and

$$
\begin{array}{r}
\left\{\frac{\partial^{2}}{\partial \tau^{2}}+\frac{\partial^{2}}{\partial \vec{x}^{2}}-m_{2}^{2}\right\}\left\langle\Xi\left|\phi^{\alpha}(\vec{x},-i \tau)\right| 0\right\rangle \\
-\lambda\left\langle\Xi\left|:\left(\chi^{2}(\vec{x},-i \tau)+\phi^{2}(\vec{x},-i \tau)\right) \phi^{\alpha}(\vec{x},-i \tau):\right| 0\right\rangle=0 .
\end{array}
$$

Due to translational invariance one has

$$
\begin{aligned}
& \left\langle\Xi\left|\chi^{a}(\vec{x},-i \tau)\right| 0\right\rangle=\Phi^{a}(\tau), \\
& \left\langle\Xi\left|\phi^{\alpha}(\vec{x},-i \tau)\right| 0\right\rangle=\Psi^{\alpha}(\tau)
\end{aligned}
$$

so that the partial derivative w.r.t. $x$ vanishes.

In the large $N$ limit one can split the matrix elements of the operators : $\left(\chi^{2}+\phi^{2}\right) \chi^{a}$ : and $:\left(\chi^{2}+\phi^{2}\right) \phi^{\alpha}:$ as follows

$$
\begin{gathered}
\left\langle a_{1} \ldots a_{n}, \beta_{1} \ldots \beta_{k}\left|:\left(\chi^{2}+\phi^{2}\right) \chi^{a}:\right| 0\right\rangle= \\
=\sum_{p, p^{\prime}} \sum_{\substack{n_{1}+n_{2}=n, k_{1}+k_{2}=k}} \frac{n ! k !}{n_{1} ! n_{2} ! k_{1} ! k_{2} !}\left\langle p\left(a_{1}\right) \ldots p\left(a_{n_{1}}\right), p^{\prime}\left(\beta_{1}\right) \ldots p^{\prime}\left(\beta_{k_{1}}\right)\left|:\left(\chi^{2}+\phi^{2}\right):\right| 0\right\rangle \times \\
\left.\times\left\langle p\left(a_{n_{1}+1}\right) \ldots p\left(a_{n}\right), p^{\prime}\left(\beta_{k_{1}+1}\right)\right) \ldots p^{\prime}\left(\beta_{k}\right)\left|\chi^{a}\right| 0\right\rangle,
\end{gathered}
$$


where $p, p^{\prime}$ stand for permutations. This formula holds for $n$ and $k$ finite as $N \rightarrow \infty$ and extends the standard factorization of $O(N)$-singlet operators at large $N$ (see e.g. Ref. 229]) to the case of multiparticle production. form

Using the definitions (3.32), (3.33), (3.34) and (3.35), we rewrite Eq. (3.42) in the

$$
\begin{array}{r}
\left\langle\Xi\left|:\left(\chi^{2}(\vec{x},-i \tau)+\phi^{2}(\vec{x},-i \tau)\right) \chi^{a}(\vec{x},-i \tau):\right| 0\right\rangle \\
=\left\langle\Xi\left|:\left(\chi^{2}(\vec{x},-i \tau)+\phi^{2}(\vec{x},-i \tau)\right):\right| 0\right\rangle\left\langle\Xi\left|\chi^{a}(\vec{x},-i \tau)\right| 0\right\rangle=v(\tau) \Phi^{a}(\tau)
\end{array}
$$

and analogously

$$
\begin{array}{r}
\left\langle\Xi\left|:\left(\chi^{2}(\vec{x},-i \tau)+\phi^{2}(\vec{x},-i \tau)\right) \phi^{\alpha}(\vec{x},-i \tau):\right| 0\right\rangle \\
=\left\langle\Xi\left|:\left(\chi^{2}(\vec{x},-i \tau)+\phi^{2}(\vec{x},-i \tau)\right):\right| 0\right\rangle\left\langle\Xi\left|\phi^{\alpha}(\vec{x},-i \tau)\right| 0\right\rangle=v(\tau) \Psi^{\alpha}(\tau)
\end{array}
$$

where translational invariance of the averages, which results in

$$
\left\langle\Xi\left|:\left(\chi^{2}(\vec{x},-i \tau)+\phi^{2}(\vec{x},-i \tau)\right):\right| 0\right\rangle=v(\tau),
$$

and Eqs. (3.40), (3.41) have been used. Substituting (3.43) and (3.44) into Eqs. (3.38) and (3.39), we obtain Eqs. (3.9) and (3.10).

To infer the remaining Eqs. (3.25), (3.26) and (3.27), let us represent $D_{\chi}^{a b}\left(\omega_{1} ; \tau, \tau^{\prime}\right)$ and $D_{\phi}^{\alpha \beta}\left(\omega_{2} ; \tau, \tau^{\prime}\right)$ given by (3.15) and $(3.16)$ as

$$
\begin{array}{r}
D_{\chi}^{a b}\left(\omega_{1} ; \tau, \tau^{\prime}\right)=\int \frac{d^{3} \vec{x}}{(2 \pi)^{3}} \mathrm{e}^{i \vec{p} \vec{x}}\left\langle\Xi\left|\mathrm{T} \chi^{a}(\vec{x},-i \tau) \chi^{b}\left(\overrightarrow{0},-i \tau^{\prime}\right)\right| 0\right\rangle \\
-\delta^{(3)}(\vec{p}) \Phi^{a}(\tau) \Phi^{b}\left(\tau^{\prime}\right)
\end{array}
$$

and

$$
\begin{array}{r}
D_{\phi}^{\alpha \beta}\left(\omega_{1} ; \tau, \tau^{\prime}\right)=\int \frac{d^{3} \vec{x}}{(2 \pi)^{3}} \mathrm{e}^{i \vec{p} \vec{x}}\left\langle\Xi\left|\mathrm{T} \phi^{\alpha}(\vec{x},-i \tau) \phi^{\beta}\left(\overrightarrow{0},-i \tau^{\prime}\right)\right| 0\right\rangle \\
-\delta^{(3)}(\vec{p}) \Psi^{\alpha}(\tau) \Psi^{\beta}\left(\tau^{\prime}\right) .
\end{array}
$$

Here the subtractions cancel disconnected parts which are not included in the definition of $D_{\chi}^{a b}$ and $D_{\phi}^{\alpha \beta}$ and T stands for the T-product. Notice that Eq. (3.27) can immediately be rewritten in the form (3.35) using (3.46) and (3.47). The terms involving the propagators $D^{a a}$ and $D^{\alpha \alpha}$ in the equation for $v(\tau)$ are associated with the connected part of the operator : $\chi^{2}+\phi^{2}:$

The operator equations $(3.36)$ and (3.37) result in the following equations for the T-products:

$$
\begin{array}{r}
\left\{\frac{\partial^{2}}{\partial \tau^{2}}+\frac{\partial^{2}}{\partial \vec{x}^{2}}-m_{1}^{2}\right\}\left\langle\Xi\left|\mathrm{T} \chi^{a}(\vec{x},-i \tau) \chi^{b}\left(\overrightarrow{0},-i \tau^{\prime}\right)\right| 0\right\rangle \\
-\lambda\left\langle\Xi\left|\mathrm{T}\left(\chi^{2}(\vec{x},-i \tau)+\phi^{2}(\vec{x},-i \tau)\right) \chi^{a}(\vec{x},-i \tau) \chi^{b}\left(\overrightarrow{0},-i \tau^{\prime}\right)\right| 0\right\rangle \\
=-\delta^{a b} \delta\left(\tau-\tau^{\prime}\right) \delta^{(3)}(\vec{x})
\end{array}
$$


and

$$
\begin{array}{r}
\left\{\frac{\partial^{2}}{\partial \tau^{2}}+\frac{\partial^{2}}{\partial \vec{x}^{2}}-m_{2}^{2}\right\}\left\langle\Xi\left|\mathrm{T} \phi^{\alpha}(\vec{x},-i \tau) \phi^{\beta}\left(\overrightarrow{0},-i \tau^{\prime}\right)\right| 0\right\rangle \\
-\lambda\left\langle\Xi\left|\mathrm{T}\left(\chi^{2}(\vec{x},-i \tau)+\phi^{2}(\vec{x},-i \tau)\right) \phi^{\alpha}(\vec{x},-i \tau) \phi^{\beta}\left(\overrightarrow{0},-i \tau^{\prime}\right)\right| 0\right\rangle \\
=-\delta^{\alpha \beta} \delta\left(\tau-\tau^{\prime}\right) \delta^{(3)}(\vec{x}),
\end{array}
$$

where the delta functions on the r.h.s.'s emerge in a standard way when differentiating the T-product and using canonical commutation relations.

Eqs. (3.48) and (3.49) are the lowest ones in the chain of the Schwinger-Dyson equations which can be extracted from the following identities

$$
\left\langle\Xi\left|\mathrm{T} \frac{\delta S[\chi, \phi]}{\delta \chi^{a}(x)} F[\chi, \phi]\right| 0\right\rangle=i\left\langle\Xi\left|\mathrm{T} \frac{\delta F[\chi, \phi]}{\delta \chi^{a}(x)}\right| 0\right\rangle
$$

and

$$
\left\langle\Xi\left|\mathrm{T} \frac{\delta S[\chi, \phi]}{\delta \phi^{\alpha}(x)} F[\chi, \phi]\right| 0\right\rangle=i\left\langle\Xi\left|\mathrm{T} \frac{\delta F[\chi, \phi]}{\delta \phi^{\alpha}(x)}\right| 0\right\rangle
$$

where $S$ is the action of the model and $F[\chi, \phi]$ is an arbitrary functional of $\chi$ and $\phi$. In particular, Eqs. (3.38) and (3.39) are associated with $F=1$ while Eqs. (3.48) and (3.49) correspond to $F=\chi^{b}\left(\overrightarrow{0},-i \tau^{\prime}\right)$ and $F=\phi^{\beta}\left(\overrightarrow{0},-i \tau^{\prime}\right)$, respectively. In the language of path integral Eqs. (3.50) and (3.51) result from the invariance of the measure under an arbitrary variation of $\chi^{a}(x)$ or $\phi^{\alpha}(x)$.

Using the large- $N$ factorization

$$
\begin{array}{r}
\left\langle\Xi\left|\mathrm{T}\left(\chi^{2}(\vec{x},-i \tau)+\phi^{2}(\vec{x},-i \tau)\right) \chi^{a}(\vec{x},-i \tau) \chi^{b}\left(\overrightarrow{0},-i \tau^{\prime}\right)\right| 0\right\rangle \\
=\left\langle\Xi\left|:\left(\chi^{2}(\vec{x},-i \tau)+\phi^{2}(\vec{x},-i \tau)\right):\right| 0\right\rangle\left\langle\Xi\left|\mathrm{T} \chi^{a}(\vec{x},-i \tau) \chi^{b}\left(\overrightarrow{0},-i \tau^{\prime}\right)\right| 0\right\rangle
\end{array}
$$

and analogously

$$
\begin{array}{r}
\left\langle\Xi\left|\mathrm{T}\left(\chi^{2}(\vec{x},-i \tau)+\phi^{2}(\vec{x},-i \tau)\right) \phi^{\alpha}(\vec{x},-i \tau) \phi^{\beta}\left(\overrightarrow{0},-i \tau^{\prime}\right)\right| 0\right\rangle \\
=\left\langle\Xi\left|:\left(\chi^{2}(\vec{x},-i \tau)+\phi^{2}(\vec{x},-i \tau)\right):\right| 0\right\rangle\left\langle\Xi\left|\mathrm{T} \phi^{\alpha}(\vec{x},-i \tau) \phi^{\beta}\left(\overrightarrow{0},-i \tau^{\prime}\right)\right| 0\right\rangle,
\end{array}
$$

as well as Eqs. (3.45), (3.9), (3.10) and upon integrating over $d^{3} x$, we obtain Eqs. (3.25), (3.26) from Eqs. (3.48), (3.49).

Some comments concerning the operator derivation of Eqs. (3.9), (3.10), (3.25), (3.26) and (3.27) are in order. The factorization was crucial to drop out the dependence on the spatial coordinates in the interaction terms and, therefore, for the reduction at large $N$ to the quantum mechanical problem which is given by the closed set of Eqs. (3.9), (3.10), (3.25), (3.26) and (3.27) Its appearance is due to the special kinematics of the produced particles whose wave functions do not depend on the spatial coordinates. The factorization formula (3.42) holds for $n$ and $k$ fixed as $N \rightarrow \infty$ (notice that only $n_{1,2} \leq n$ and $k_{1,2} \leq k$ enter Eq. (3.42)). The corrections to the large- $N$ amplitudes are known [12, 15] to be 
controlled at large $n$ by the parameter $n^{2} \lambda \sim n^{2} / N$ (also by $k^{2} / N$ in the given case) and are due to rescatterings of the produced particles. Hence, equations (3.9), (3.10), (3.25), (3.26) and (3.27) allow for the calculation of the multiparticle amplitudes only for $n, k \ll \sqrt{N}$.

\section{The exact solution}

To solve the set of equations (3.9), (3.10), (3.25), (3.26) and (3.27), let us first look at Eqs. (3.25) and (3.26) for a given $v(\tau)$. These equations determine the Green functions of the Schrödinger operator with the potential $v(\tau)$ while $\tau$ plays the role of a 1-dimensional coordinate. Equivalently $G^{T}\left(\omega ; \tau, \tau^{\prime}\right)$ is the matrix element of the resolvent

$$
G^{T}\left(\omega ; \tau, \tau^{\prime}\right)=\left\langle\tau\left|\frac{1}{-D^{2}+\omega^{2}+v}\right| \tau^{\prime}\right\rangle
$$

where $D$ stands for $d / d \tau$ for brevity. One should then take the diagonal matrix element of the resolvent, $G^{T}(\omega ; \tau, \tau)$, to substitute it into Eq. (3.27) and determine $v(\tau)$ versus $\Phi^{2}$ and $\Psi^{2}$.

The general solution of this problem for arbitrary $v$ is given by the Gelfand-Dikiu formula 30

$$
G^{T}(\omega ; \tau, \tau)=R_{\omega}[v] \equiv \sum_{l=0}^{\infty} \frac{R_{l}[v]}{\omega^{2 l+1}}
$$

where the differential polynomials $R_{l}[v]$ are determined recurrently by

$$
R_{l}[v]=\frac{1}{2^{l}}\left(\frac{1}{2} D^{2}-v-D^{-1} v D\right)^{l} \cdot \frac{1}{2}
$$

and the inverse operator is

$$
D^{-1} v(\tau)=\int_{-\infty}^{\tau} d x v(x)
$$

Eq. (4.3) stems from the fact that $R_{\omega}[v]$ obeys the third order linear differential equation

$$
\frac{1}{2}\left(\frac{1}{2} D^{3}-D v-v D\right) R_{\omega}[v]=\omega^{2} D R_{\omega}[v] .
$$

The polynomials $R_{l}[v]$ depend on $v$ and its derivatives $v^{(s)} \equiv\left(D^{s} v\right)$. The first few polynomials are

$$
\begin{array}{r}
R_{0}[v]=\frac{1}{2}, \quad R_{1}[v]=-\frac{v}{4}, \quad R_{2}[v]=\frac{1}{16}\left(3 v^{2}-v^{\prime \prime}\right) \\
R_{3}[v]=-\frac{1}{64}\left(10 v^{3}-10 v v^{\prime \prime}-5\left(v^{\prime}\right)^{2}+v^{(4)}\right), \ldots
\end{array}
$$

while for $\tau$-independent $v(\tau)=v_{0}$ one has

$$
R_{\omega}\left[v_{0}\right]=\frac{1}{2 \sqrt{\omega^{2}+v_{0}}} \quad\left(v_{0}=\text { const. }\right)
$$


which agrees with Eq. (3.18) at $\tau=\tau^{\prime}$.

Following Ref. [26] we renormalize the above equations. Let us introduce $v_{0}$ as

$$
v_{0}=\frac{\lambda}{4 \pi^{2}}\left(N_{1} \int_{m_{1 R}} d \omega \sqrt{\omega^{2}-m_{1 R}^{2}}+N_{2} \int_{m_{2 R}} d \omega \sqrt{\omega^{2}-m_{2 R}^{2}}\right),
$$

where the renormalized masses $m_{1,2 R}$ are defined by the following equations

$$
m_{1 R}^{2}=m_{1}^{2}+v_{0}, \quad m_{2 R}^{2}=m_{2}^{2}+v_{0} .
$$

We also introduce the renormalized coupling constant defined as

$$
\frac{1}{\lambda_{R}}=\frac{1}{\lambda}+\frac{N_{1}}{8 \pi^{2}} \int_{m_{1 R}} d \omega \frac{\sqrt{\omega^{2}-m_{1 R}^{2}}}{\omega^{2}}+\frac{N_{2}}{8 \pi^{2}} \int_{m_{2 R}} d \omega \frac{\sqrt{\omega^{2}-m_{2 R}^{2}}}{\omega^{2}} .
$$

The renormalized potential $v_{R}(\tau)$ reads

$$
\begin{gathered}
v_{R}(\tau)=v(\tau)-v_{0}= \\
=\lambda_{R}\left(\Phi^{2}+\Psi^{2}\right)+\frac{\lambda_{R}}{2 \pi^{2}}\left[N_{1} \int_{m_{1 R}} d \omega \omega \sqrt{\omega^{2}-m_{1 R}^{2}}\left(R_{\omega}\left[v_{R}\right]-\frac{R_{1}\left[v_{R}\right]}{\omega^{3}}-\frac{1}{2 \omega}\right)+\right. \\
\left.+N_{2} \int_{m_{2 R}} d \omega \omega \sqrt{\omega^{2}-m_{2 R}^{2}}\left(R_{\omega}\left[v_{R}\right]-\frac{R_{1}\left[v_{R}\right]}{\omega^{3}}-\frac{1}{2 \omega}\right)\right] .
\end{gathered}
$$

Now everything is expressed in terms of $m_{1,2 R}$ and $v_{R}(\tau)$ :

$$
\begin{gathered}
\left\{D^{2}-m_{1 R}^{2}-v_{R}(\tau)\right\} \Phi^{a}(\tau)=0, \\
\left\{D^{2}-m_{2 R}^{2}-v_{R}(\tau)\right\} \Psi^{a}(\tau)=0, \\
\omega=\sqrt{\vec{p}^{2}+m_{1,2 R}^{2}} .
\end{gathered}
$$

The integrals over $\omega$ on the r.h.s. become convergent after the renormalizations. The meaning of renormalization is that one chooses the bare quantities, $m_{1,2}^{2}$ and $\lambda$, to be dependent on the cut-off according to Eqs. (4.9) and (4.10) and render the renormalized ones, $m_{R}^{2}$ and $\lambda_{R}$, cut-off-independent.

We can easily find an exact solution to these equations. Let us denote

$$
L=\frac{1}{2}\left(\frac{1}{2} D^{3}-D v-v D\right) .
$$

We shall look for $R_{\omega}\left[v_{R}\right]$ in the following form

$$
R_{\omega}\left[v_{R}\right]=a(\omega)+x(\omega) \Phi^{2}+y(\omega) \Psi^{2},
$$

where $a, x$ and $y$ are constants which may depend on $\omega$. Using eq. (4.5) and the fact [26] that $\Phi^{2}$ and $\Psi^{2}$ are the eigenvectorsf of the operator $D^{-1} L$ :

$\frac{1}{2}\left(\frac{1}{2} D^{2}-v_{R}-D^{-1} v_{R} D\right) \Phi^{2}=\frac{1}{2} D^{-1}\left(\frac{1}{2} D^{3}-D v_{R}-v_{R} D\right) \Phi^{2}=D^{-1} D m_{1 R}^{2} \Phi^{2}=m_{1 R}^{2} \Phi^{2}$,

\footnotetext{
${ }^{5}$ This is derived using solely Eqs. (4.12) and (4.13) for an arbitrary $v_{R}(\tau)$ and the fact that $\Phi^{2}(\tau)$ and $\Psi^{2}(\tau)$ vanish for $\tau \rightarrow-\infty$.
} 
$\frac{1}{2}\left(\frac{1}{2} D^{2}-v_{R}-D^{-1} v_{R} D\right) \Psi^{2}=\frac{1}{2} D^{-1}\left(\frac{1}{2} D^{3}-D v_{R}-v_{R} D\right) \Psi^{2}=D^{-1} D m_{2 R}^{2} \Phi^{2}=m_{2 R}^{2} \Psi^{2}$,

one gets

$$
\begin{aligned}
L R_{\omega}\left[v_{R}\right]= & -\frac{a}{2} D v_{R}+x m_{1 R}^{2} D \Phi^{2}+y m_{2 R}^{2} D \Psi^{2}= \\
& =\omega^{2} D\left(a+x \Phi^{2}+y \Psi^{2}\right) .
\end{aligned}
$$

We remove $D$ from both sides of the above equation and we get

$$
v_{R}=c+\frac{2 x}{a}\left(m_{1 R}^{2}-\omega^{2}\right) \Phi^{2}+\frac{2 y}{a}\left(m_{2 R}^{2}-\omega^{2}\right) \Psi^{2},
$$

where $c$ is a constant of integration. Thus we have

$$
v_{R}=c+\alpha \Phi^{2}+\beta \Psi^{2}
$$

where the constants

$$
\alpha=-\frac{2 x\left(\omega^{2}-m_{1 R}^{2}\right)}{a}, \quad \beta=-\frac{2 y\left(\omega^{2}-m_{2 R}^{2}\right)}{a}
$$

do not depend on $\omega$ since $v_{R}$ does not.

We now substitute both expressions for $R_{\omega}$ and $v_{R}$ into the equation (4.11) which determines $v_{R}$. One can easily see that $c=0, a=1 /(2 \omega)$ (notice that this value of $a$ agrees with the perturbation expansion) and

$$
\begin{aligned}
& \alpha=\frac{\lambda_{R}}{1+\frac{\lambda_{R}}{8 \pi^{2}} I_{1}}, \quad x=-\frac{\alpha}{4 \omega\left(\omega^{2}-m_{1 R}^{2}\right)}, \\
& \beta=\frac{\lambda_{R}}{1+\frac{\lambda_{R}}{8 \pi^{2}} I_{2}}, \quad y=-\frac{\beta}{4 \omega\left(\omega^{2}-m_{2 R}^{2}\right)},
\end{aligned}
$$

where

$$
\begin{aligned}
I_{1}= & N_{1} \int_{m_{1 R}} d \omega \sqrt{\omega^{2}-m_{1 R}^{2}}\left[\frac{1}{\omega^{2}-m_{1 R}^{2}}-\frac{1}{\omega^{2}}\right]+ \\
& +N_{2} \int_{m_{2 R}} d \omega \sqrt{\omega^{2}-m_{2 R}^{2}}\left[\frac{1}{\omega^{2}-m_{1 R}^{2}}-\frac{1}{\omega^{2}}\right], \\
I_{2}= & N_{1} \int_{m_{1 R}} d \omega \sqrt{\omega^{2}-m_{1 R}^{2}}\left[\frac{1}{\omega^{2}-m_{2 R}^{2}}-\frac{1}{\omega^{2}}\right]+ \\
& +N_{2} \int_{m_{2 R}} d \omega \sqrt{\omega^{2}-m_{2 R}^{2}}\left[\frac{1}{\omega^{2}-m_{2 R}^{2}}-\frac{1}{\omega^{2}}\right] .
\end{aligned}
$$

After some calculations one gets

$$
I_{1}=N_{1}+N_{2} \int_{m_{2 R}} d \omega \sqrt{\omega^{2}-m_{2 R}^{2}}\left[\frac{1}{\omega^{2}-m_{1 R}^{2}}-\frac{1}{\omega^{2}}\right]
$$


and

$$
I_{2}=N_{2}+N_{1} \int_{m_{1 R}} d \omega \sqrt{\omega^{2}-m_{1 R}^{2}}\left[\frac{1}{\omega^{2}-m_{2 R}^{2}}-\frac{1}{\omega^{2}}\right] .
$$

For $m_{1}=m_{2}$ these formulas recover the results [26] for the $O\left(N_{1}+N_{2}\right)$-case. The fact that the solution is unique can be proven quite similarly to Ref. [26].

The diagonal resolvent reads

$$
R_{\omega}\left[v_{R}\right]=\frac{1}{2 \omega}-\frac{\alpha \Phi^{2}}{4 \omega\left(\omega^{2}-m_{1 R}^{2}\right)}-\frac{\beta \Psi^{2}}{4 \omega\left(\omega^{2}-m_{2 R}^{2}\right)} .
$$

The appearance of the poles in the resolvent has two faces.

1. From one point of view it is not directly related to the propagation of particles in the loops. For example the pole in the resolvent appears for the symmetric $O(N)$ case even at the tree level. The pole is actually due to an existence of a strong classical external field which induces a potential $v(\tau)$ and corresponds to a bound state of a quantum mechanical particle. Thus the pole in the resolvent is actually a manifestation of a bound state in the spectrum of the corresponding Schrödinger operator. This is of course an obvious fact but it explains the appearance of an extra pole when we switch on an additional particle. Emissions of an additional particle induce a new term in the effective potential which is not suppressed at large $N$ since we assume that the wave function of an emitted particle is of order $\sqrt{N}$. This new potential is responsible for a new bound state of the quantum mechanical particle. This also explains why the residue of the additional pole in the resolvent is not suppressed at large $N$.

A natural question would be as to why the poles should be exactly at the values of the masses of the particles (a priori these poles could be anywhere). The answer is simple: this is because we know that there exist solutions [23] to the Schrödinger equation for $\omega=m_{1,2}$ which decrease at both infinities. Thus we know that indeed they are the bound states with the energies $m_{1,2}$.

2. On the other hand the diagonal resolvent itself corresponds to a loop by definition since it corresponds to propagator integrated over the energy (i.e. to the one with coinciding ends in the $\tau$-space). For simplicity let us consider the model of the $O\left(N_{1}\right)$-particle with the mass $m_{1}$ and an additional singlet one with the mass $m_{2}$, i.e. $N_{2}=1$. In perturbation theory the simplest correction to the propagator of the $O\left(N_{1}\right)$-particle is given by a diagram with a 4 -vertex which produces 2 outgoing particles with the mass $m_{2}$. To extract the diagonal resolvent we should make a loop of this propagator which is done in the momentum space by integrating over the energy $(d \epsilon)$ of the virtual particle. Thus we easily see that the correction to the resolvent which is due to an emission of two particles is proportional to

$$
R_{\omega}^{(2)} \propto \int_{-\infty}^{\infty} \frac{d \epsilon}{2 \pi} \frac{1}{\left(\omega^{2}-\epsilon^{2}-i 0\right)\left(\omega^{2}-\left(\epsilon-2 m_{2}\right)^{2}-i 0\right)},
$$

where $\omega^{2}=|p|^{2}+m_{1}^{2}$, and $\vec{p}$ is a spatial virtual momentum. This integral is convergent and can be computed by closing the contour down around $\epsilon=\omega-i 0$ and $\epsilon=\omega-2 m_{2}-i 0$. 
It is easy to see that a pole appears at $\omega=m_{2}$ :

$$
R_{\omega}^{(2)} \propto \frac{i}{4 m_{2} \omega\left(\omega-m_{2}-i 0\right)} .
$$

This is exactly what is implied by (4.29).

We thus have checked that there should be two poles in the resolvent in the model of two particles with different masses. These poles sit exactly at the masses of those particles.

We now can find the expressions for $\Phi^{a}$ and $\Psi^{\alpha}$. We have the following equations

$$
\begin{aligned}
& \left\{D^{2}-m_{1 R}^{2}-\left(\alpha \Phi^{2}+\beta \Psi^{2}\right)\right\} \Phi^{a}=0, \\
& \left\{D^{2}-m_{2 R}^{2}-\left(\alpha \Phi^{2}+\beta \Psi^{2}\right)\right\} \Psi^{\alpha}=0 .
\end{aligned}
$$

These equations reduce to the classical ones (with the renormalized coupling constant $\lambda_{R}$ ) if we renormalize the fields $\Phi^{a}$ and $\Psi^{\alpha}$ as follows

$$
\tilde{\Phi}^{a}=\sqrt{\frac{\alpha}{\lambda_{R}}} \Phi^{a}=\left(1+\frac{\lambda_{R}}{8 \pi^{2}} I_{1}\right)^{-1 / 2} \Phi^{a}, \quad \tilde{\Psi}^{\alpha}=\sqrt{\frac{\beta}{\lambda_{R}}} \Psi^{\alpha}=\left(1+\frac{\lambda_{R}}{8 \pi^{2}} I_{2}\right)^{-1 / 2} \Psi^{\alpha} .
$$

The solution to these equations is given by the expressions which are a straightforward generalization of those found in Ref. [23]:

$$
\begin{aligned}
& \tilde{\Phi}^{a}=\tilde{z}_{1}^{a}\left(1-2 \lambda_{R} \frac{k}{m_{2 R}^{2}} \tilde{z}_{2}^{2}\right)\left(1-\frac{2 \lambda_{R}}{m_{1 R}^{2}} \tilde{z}_{1}^{2}-\frac{2 \lambda_{R}}{m_{2 R}^{2}} \tilde{z}_{2}^{2}+\lambda_{R}^{2} \frac{k^{2}}{m_{1 R}^{2} m_{2 R}^{2}} \tilde{z}_{1}^{2} \tilde{z}_{2}^{2}\right)^{-1}, \\
& \tilde{\Psi}^{\beta}=\tilde{z}_{2}^{\beta}\left(1+2 \lambda_{R} \frac{k}{m_{1 R}^{2}} \tilde{z}_{1}^{2}\right)\left(1-\frac{2 \lambda_{R}}{m_{1 R}^{2}} \tilde{z}_{1}^{2}-\frac{2 \lambda_{R}}{m_{2 R}^{2}} \tilde{z}_{2}^{2}+\lambda_{R}^{2} \frac{k^{2}}{m_{1 R}^{2} m_{2 R}^{2}} \tilde{z}_{1}^{2} \tilde{z}_{2}^{2}\right)^{-1},
\end{aligned}
$$

where

$$
k=\frac{m_{1 R}-m_{2 R}}{m_{1 R}+m_{2 R}}
$$

and

$$
\begin{aligned}
& \tilde{z}_{1}^{a}=\xi_{1}^{a} \sqrt{\frac{\alpha}{\lambda_{R}}} e^{m_{1 R} \tau}=\xi_{1}^{a} e^{m_{1 R} \tau}\left(1+\frac{\lambda_{R}}{8 \pi^{2}} I_{1}\right)^{-1 / 2} \\
& \tilde{z}_{2}^{\alpha}=\xi_{2}^{\alpha} \sqrt{\frac{\beta}{\lambda_{R}}} e^{m_{2 R} \tau}=\xi_{2}^{\alpha} e^{m_{2 R} \tau}\left(1+\frac{\lambda_{R}}{8 \pi^{2}} I_{2}\right)^{-1 / 2}
\end{aligned}
$$

Notice that the constants $\alpha$ and $\beta$ are in general complex. By assuming for definiteness that $m_{2 R}^{2} \geq m_{1 R}^{2}$ one can easily see that the constant $\alpha$ is real while the imaginary part of $\beta$ is given by the following equation

$$
\operatorname{Im} \frac{1}{\beta}=-\frac{N_{1}}{8 \pi} \cdot \frac{\sqrt{m_{2 R}^{2}-m_{1 R}^{2}}}{2 m_{2 R}} .
$$




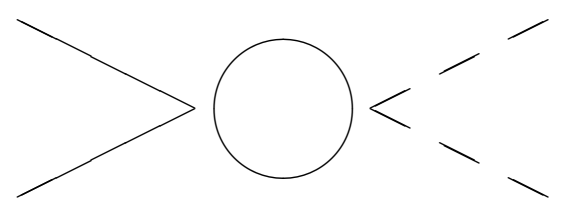

Figure 5: The one-loop diagram for the process $\chi+\chi \rightarrow \phi+\phi$ which possesses an imaginary part for $m_{2 R}>m_{1 R}$. given by Eq. (4.40).

The appearance of this imaginary part is in contrast to the $O(N)$ model with unbroken symmetry where the amplitudes are real [26. In the present model the imaginary part appears in $\alpha$ or $\beta$ because the masses of $O\left(N_{1}\right)$ and $O\left(N_{2}\right)$ particles are not equal. Indeed in a particular example $m_{2 R}^{2} \geq m_{1 R}^{2}$ an imaginary part appears in the amplitude of scattering of two $\chi$ particles due to a possibility of an inelastic process $\chi+\chi \rightarrow \phi+\phi$. This imaginary part is associated with the diagram of Fig. 5. A direct calculation of the imaginary part of the diagram of Fig. 5 gives the same expression (4.40).

In the case of $N_{2}=1$ which is discussed above, we drop out the contributions proportional to $D_{\phi}^{\alpha \alpha}$ in the recurrence equations. Therefore this case can be easily recovered from Eqs. (4.35) and (4.36) by taking formally $N_{2}=0$ in Eqs. (4.27) and (4.28) for $I_{1}$ and $I_{2}$ which enter the expressions (4.23) and (4.24) for the constants $\alpha$ and $\beta$. The correct imaginary part immediately appears for $m_{2} \geq m_{1}$ after integrating (4.31) over $\omega$. This imaginary part corresponds to on-mass-shell virtual $O\left(N_{1}\right)$ particles emitting heavier singlet particles (the wave functions for incoming particles are factorized out from this diagram and therefore it can be thought as a contribution for the forward scattering for singlet particles).

A generalization of the equations for the generating functions $\Phi, \Psi$ to the case of $O\left(N_{1}\right) \times \ldots \times O\left(N_{s}\right)$ scalar field is straightforward ( $s$ is a positive integer). In such a general case which is described by the Lagrangian

$$
\mathcal{L}=\frac{1}{2} \sum_{i=1}^{s}\left(\partial_{\mu} \phi_{i}\right)\left(\partial_{\mu} \phi_{i}\right)-\frac{1}{2} \sum_{i=1}^{s} m_{i}^{2} \phi_{i}^{2}-\frac{\lambda}{4}\left(\sum_{i=1}^{s} \phi_{i}^{2}\right)^{2}
$$

the equations for the generating functions for the amplitudes of the multiparticle production at the threshold by a single particle are given by equations similar to the classical ones while the effect of loops in the large $N$ is reduced to a renormalization of the masses and the coupling constant and to a (complex) renormalization of the wave functions $\xi_{i}$ of the external particles.

The potential of the associated quantum mechanical problem is

$$
v_{R}=\sum_{i} \alpha_{i} \Phi_{i}^{2},
$$


where $\Phi_{i}^{a}$ are the generating functions for the amplitudes of multiparticle production by a single particle $\phi_{i}^{a}$, and the parameters $\alpha_{i}$ are defined as follows

$$
\frac{1}{\alpha_{i}}=\frac{1}{\lambda_{R}}+\frac{1}{8 \pi^{2}} \sum_{j} N_{j} \int_{m_{j R}} d \omega \sqrt{\omega^{2}-m_{j R}^{2}}\left[\frac{1}{\omega^{2}-m_{i R}^{2}}-\frac{1}{\omega^{2}}\right] .
$$

Here $m_{i R}$ stand for a renormalized mass of the $i$-particle. The diagonal resolvent reads

$$
R_{\omega}\left[v_{R}\right]=\frac{1}{2 \omega}-\sum_{i} \frac{\alpha_{i} \Phi_{i}^{2}}{4 \omega\left(\omega^{2}-m_{i R}^{2}\right)}
$$

This resolvent corresponds to a nullification of all amplitudes except for those associated with the production of two identical particles.

\section{A model with spontaneously broken symmetry}

\subsection{The Schwinger-Dyson equations}

Let us now consider the model of an $O\left(N_{1}\right)$ scalar field and a singlet scalar field with the wrong sign of the mass square. The Lagrangian reads

$$
\mathcal{L}=\frac{1}{2}\left(\partial_{\mu} \chi^{b}\right)\left(\partial_{\mu} \chi^{b}\right)+\frac{1}{2}\left(\partial_{\mu} \phi\right)\left(\partial_{\mu} \phi\right)-\frac{1}{2} m_{1}^{2}\left(\chi^{b} \chi^{b}\right)-\frac{1}{2} m_{2}^{2}\left(\phi^{2}\right)-\frac{1}{4} \lambda\left(\phi \phi+\chi^{b} \chi^{b}\right)^{2},
$$

where $m_{2}^{2}<0$ and $m_{1}^{2} \geq-\left|m_{2}^{2}\right|$ can be either positive or negative fo The interaction term has an $O(N)$-symmetry with $N=N_{1}+1$ which is explicitly broken for $m_{1}^{2} \neq m_{2}^{2}$ by the mass term. The Lagrangian (5.1) is invariant for $m_{1}^{2} \neq m_{2}^{2}$ under the reflection: $\phi \rightarrow-\phi$.

At the classical level one can see that the reflection symmetry is spontaneously broken and the field $\phi$ acquires a vacuum expectation value

$$
\phi_{0}=\frac{\left|m_{2}\right|}{\sqrt{\lambda}}
$$

while the masses of the physical particles are

$$
m_{\chi}=\sqrt{m_{1}^{2}+\left|m_{2}^{2}\right|}, \quad m_{\phi}=\sqrt{2}\left|m_{2}\right| \quad \text { (tree level) } .
$$

If the $O(N)$-symmetry were not broken explicitly by the mass term (i.e. if $m_{1}=m_{2}$ ), one would get $N-1$ Goldstone bosons with the mass $m_{\chi}=0$.

The classical equations of motion read

$$
\begin{aligned}
& \left\{\partial_{\mu}^{2}+m_{1}^{2}+\lambda\left(\chi^{a} \chi^{a}+\phi^{2}\right)\right\} \chi^{b}=0, \\
& \left\{\partial_{\mu}^{2}-\left|m_{2}^{2}\right|+\lambda\left(\chi^{a} \chi^{a}+\phi^{2}\right)\right\} \phi=0 .
\end{aligned}
$$

\footnotetext{
${ }^{6}$ Notice that in this case the vacuum state corresponds to an unbroken $O\left(N_{1}\right)$ symmetry.
} 
At the classical level one can shift the field $\phi=\phi^{\prime}+\phi_{0}$. Then the classical equations of motion take the form

$$
\begin{array}{r}
\left\{\partial_{\mu}^{2}+\left(m_{1}^{2}+\left|m_{2}^{2}\right|\right)+2 \lambda \phi_{0} \phi^{\prime}+\lambda\left(\chi^{a} \chi^{a}+\phi^{2}\right)\right\} \chi^{b}=0, \\
\partial_{\mu}^{2} \phi^{\prime}+2\left|m_{2}^{2}\right| \phi^{\prime}+3 \lambda \phi_{0} \phi^{\prime 2}+\lambda \phi_{0} \chi^{a} \chi^{a}+\lambda\left(\chi^{a} \chi^{a}+\phi^{\prime 2}\right) \phi^{\prime}=0 .
\end{array}
$$

One would expect that Eqs. (5.5) get simply generalized to the quantum level through the substitution of a normal ordering into (5.5). However this is not quite correct because the vacuum expectation value $\phi_{0}$ can be renormalized due to the $\phi^{\prime} \chi^{2}$ interaction. Therefore it is convenient to consider the equations of motion for the unshifted field $\phi$.

One can extract the equations for the amplitudes to produce physical particles by using the same method with the case of no spontaneous symmetry breaking. We shall assume that the field $\phi$ contains a constant part which corresponds to a correct (at the quantum level) vacuum expectation value of $\phi$. We then take the matrix elements which are associated with the production of physical particles at the threshold when the wave functions of the produced particles are translationally invariant similar to the procedure followed for the case without spontaneous breaking of the $O(N)$ symmetry.

Indeed we have the following operator equations

$$
\left(\partial_{\mu} \partial_{\mu}+m_{1}^{2}\right) \chi^{a}+\lambda:\left(\chi^{2}+\phi^{2}\right) \chi^{a}:=0
$$

and

$$
\left(\partial_{\mu} \partial_{\mu}-\left|m_{2}^{2}\right|\right) \phi+\lambda:\left(\chi^{2}+\phi^{2}\right) \phi:=0,
$$

where : : stands for a normal odering. Let us take the matrix elements of these equations between the state $\left\langle a_{1} \ldots a_{n}, \phi^{\prime k}\right|$ and the vacuum where $\phi^{\prime}$ stands for a physical particle that corresponds to the field $\phi$.

One can see that

$$
\Phi^{a}(\tau)=\sum_{n, k \geq 1} \frac{1}{n ! k !}\left\langle a_{1} \ldots a_{n}, \phi^{\prime k}\left|\chi^{a}(\overrightarrow{0},-i \tau)\right| 0\right\rangle \xi_{1}^{b_{1}} \ldots \xi_{1}^{b_{n}} \xi_{2}^{k}
$$

and

$$
\Psi(\tau)=\sum_{n, k \geq 1} \frac{1}{n ! k !}\left\langle a_{1} \ldots a_{n}, \phi^{\prime k}|\phi(\overrightarrow{0},-i \tau)| 0\right\rangle \xi_{1}^{b_{1}} \ldots \xi_{1}^{b_{n}} \xi_{2}^{k}
$$

are nothing but the generating functions for the multiparticle production from a single $\chi$ or $\phi^{\prime}$ particle, respectively. By introducing again the coherent state

$$
\left\langle\Xi^{\prime}\right|=\sum_{n, k \geq 1} \frac{1}{n ! k !} \xi_{1}^{b_{1}} \ldots \xi_{1}^{b_{n}} \xi_{2}^{k}\left\langle a_{1} \ldots a_{n}, \phi^{\prime k}\right|
$$

we rewrite Eqs. (5.8) and (5.9) as

$$
\Phi^{a}(\tau)=\left\langle\Xi^{\prime}\left|\chi^{a}(\overrightarrow{0},-i \tau)\right| 0\right\rangle
$$




$$
\Psi(\tau)=\left\langle\Xi^{\prime}|\phi(\overrightarrow{0},-i \tau)| 0\right\rangle
$$

In the large $N$ limit one can split the operators $:\left(\chi^{2}+\phi^{2}\right) \chi^{a}:$ and $:\left(\chi^{2}+\phi^{2}\right) \phi:$ as follows

$$
\begin{gathered}
\left\langle a_{1} \ldots a_{n}, \phi^{\prime k}\left|:\left(\chi^{2}+\phi^{2}\right) \chi^{a}:\right| 0\right\rangle= \\
=\sum_{p} \sum_{\substack{n_{1}+n_{2}=n, k_{1}+k_{2}=k}} \frac{n ! k !}{n_{1} ! n_{2} ! k_{1} ! k_{2} !}\left\langle p\left(a_{1}\right) \ldots p\left(a_{n_{1}}\right), \phi^{\prime k_{1}}\left|:\left(\chi^{2}+\phi^{2}\right):\right| 0\right\rangle \times \\
\times\left\langle p\left(a_{n_{1}+1}\right) \ldots p\left(a_{n}\right), \phi^{\prime k_{2}}\left|\chi^{a}\right| 0\right\rangle
\end{gathered}
$$

and

$$
\begin{gathered}
\left\langle a_{1} \ldots a_{n}, \phi^{\prime k}\left|:\left(\chi^{2}+\phi^{2}\right) \phi:\right| 0\right\rangle= \\
=\sum_{p} \sum_{\substack{n_{1}+n_{2}=n, k_{1}+k_{2}=k}} \frac{n ! k !}{n_{1} ! n_{2} ! k_{1} ! k_{2} !}\left\langle p\left(a_{1}\right) \ldots p\left(a_{n_{1}}\right), \phi^{\prime k_{1}}\left|:\left(\chi^{2}+\phi^{2}\right):\right| 0\right\rangle \times \\
\times\left\langle p\left(a_{n_{1}+1}\right) \ldots p\left(a_{n}\right), \phi^{\prime k_{2}}|\phi| 0\right\rangle
\end{gathered}
$$

where $p$ stand for permutations. Using the definition (5.10), Eqs. (5.13) and (5.14) can be rewritten in the simple form

$$
\left\langle\Xi^{\prime}\left|:\left(\chi^{2}+\phi^{2}\right) \chi^{a}:\right| 0\right\rangle=\left\langle\Xi^{\prime}\left|:\left(\chi^{2}+\phi^{2}\right):\right| 0\right\rangle\left\langle\Xi^{\prime}\left|\chi^{a}\right| 0\right\rangle
$$

and

$$
\left\langle\Xi^{\prime}\left|:\left(\chi^{2}+\phi^{2}\right) \phi:\right| 0\right\rangle=\left\langle\Xi^{\prime}\left|:\left(\chi^{2}+\phi^{2}\right):\right| 0\right\rangle\left\langle\Xi^{\prime}|\phi| 0\right\rangle
$$

The Schwinger-Dyson equations can now be derived quite similarly to Subsect. 3.2. It is crucial again that one drops out the dependence on $3 \mathrm{D}$ spatial coordinates in the interaction terms. This is correct due to the factorization of these terms in the large- $N$ limit and the special kinematics of the produced particles. We thus get the following equations

$$
\begin{gathered}
\left\{D^{2}-m_{\chi}^{2}-v(\tau)\right\} \chi^{a}=0, \\
\left\{D^{2}-v(\tau)\right\} \phi=0
\end{gathered}
$$

where

$$
v(\tau)=\lambda\left(\Phi^{2}+\Psi^{2}-\phi_{0}^{2}\right)+\lambda \int \frac{d^{4} p}{(2 \pi)^{4}} D_{\chi}^{b b}(\tau ; p) .
$$

Here we have taken into account that $\left|m_{2}^{2}\right|=\lambda \phi_{0}^{2}$ and used the physical mass $m_{\chi}$ of the field $\chi$ which appears when we take into account the non-vanishing vacuum expectation value of the field $\phi$. At the classical level $m_{\chi}$ is given by Eq. (5.3).

The propagator $D_{\chi}^{a b}$ obeys Eq. (3.11) as for the case without spontaneous breaking. Introducing $D_{\chi}^{a b}\left(\omega ; \tau, \tau^{\prime}\right)$ (the mixed representation) as in Eq. (3.15) obeying

$$
\left\{D^{2}-\omega^{2}-v(\tau)\right\} \frac{1}{N} D_{\chi}^{b b}\left(\omega ; \tau, \tau^{\prime}\right)=-\delta\left(\tau-\tau^{\prime}\right)
$$


where $\omega^{2}=m_{\chi}^{2}+|\vec{p}|^{2}$ ( $\vec{p}$ stands for the spatial components of momentum), we can rewrite the potential $v(\tau)$ given by (5.19) as follows

$$
v(\tau)=\lambda\left(\Phi^{2}+\Psi^{2}-\phi_{0}^{2}\right)+\frac{\lambda N}{2 \pi^{2}} \int_{m_{\chi}} d \omega \omega \sqrt{\omega^{2}-m_{\chi}^{2}} G^{T}(\omega ; \tau, \tau),
$$

where $G^{T}$ stands for the tensor part of $D_{\chi}^{a b}$. Actually these equations can be easily obtained through the use of generalized recurrence equations for the amplitudes to produce particles $\chi^{a}$ and $\phi^{\prime}$. To do that we have to use the Lagrangian for the fields $\chi$ and $\phi^{\prime}=\phi-\phi_{0}$ which are physical at the classical level. The only difference with the case of no spontaneous breaking is a presence of the vertices $\chi^{2} \phi^{\prime}$ and $\phi^{\prime 3}$.

We now renormalize the masses and the coupling constant. Let us introduce

$$
v_{0}=\frac{\lambda N}{4 \pi^{2}} \int_{m_{\chi}} d \omega \sqrt{\omega^{2}-m_{\chi}^{2}}
$$

The renormalized masses are then defined as follows:

$$
m_{1 R}^{2}=m_{1}^{2}+v_{0}, \quad m_{2 R}^{2}=m_{2}^{2}+v_{0}
$$

while for the renormalized coupling constant we have

$$
\frac{1}{\lambda_{R}}=\frac{1}{\lambda}+\frac{N}{8 \pi^{2}} \int_{m_{\chi}} d \omega \frac{\sqrt{\omega^{2}-m_{\chi}^{2}}}{\omega^{2}} .
$$

We see that the physical mass of the field $\chi$ is not renormalized, $m_{\chi}^{2}=m_{1 R}^{2}-m_{2 R}^{2}=m_{\chi}^{2}$.

The renormalized potential is defined as

$$
\begin{gathered}
v_{R}(\tau)=v(\tau)-v_{0}= \\
=\lambda_{R}\left(\Phi^{2}+\Psi^{2}-\phi_{0}^{2}\right)+\frac{\lambda_{R}}{2 \pi^{2}} N_{1} \int_{m_{1 R}} d \omega \omega \sqrt{\omega^{2}-m_{1 R}^{2}}\left(R_{\omega}\left[v_{R}\right]-\frac{R_{1}\left[v_{R}\right]}{\omega^{3}}-\frac{1}{2 \omega}\right) .
\end{gathered}
$$

Here $\phi_{0}=\left|m_{2 R}\right| / \sqrt{\lambda_{R}}$ is the renormalized vacuum expectation value of the field $\phi$.

Now everything is expressed in terms of $m_{1,2 R}$ and $v_{R}(\tau)$ :

$$
\begin{gathered}
\left\{D^{2}-m_{\chi}^{2}-v_{R}(\tau)\right\} \Phi^{a}(\tau)=0, \\
\omega=\sqrt{\vec{p}^{2}+m_{\chi}^{2}} .
\end{gathered}
$$

The integral over $\omega$ on the r.h.s. of Eq. (5.25) becomes convergent after the renormalizations. 


\subsection{The exact solution}

We shall look for $R_{\omega}\left[v_{R}\right]$ in the following form

$$
R_{\omega}\left[v_{R}\right]=a(\omega)+x(\omega) \Phi^{2}+y(\omega) \Psi^{2}
$$

where $a, x$ and $y$ are constants which may depend on $\omega$. Similarly with the previous case of no spontaneous breaking we shall use Eq. (4.5) and the fact that

$$
\begin{gathered}
L \Phi^{2}=\frac{1}{2}\left(\frac{1}{2} D^{3}-D v_{R}-v_{R} D\right) \Phi^{2}=m_{\chi}^{2} D \Phi^{2}, \\
L \Psi^{2}=\frac{1}{2}\left(\frac{1}{2} D^{3}-D v_{R}-v_{R} D\right) \Psi^{2}=0 .
\end{gathered}
$$

We obtain

$$
\begin{gathered}
L R_{\omega}\left[v_{R}\right]=-\frac{a}{2} D v_{R}+x m_{\chi}^{2} D \Phi^{2}= \\
=\omega^{2} D\left(a+x \Phi^{2}+y \Psi^{2}\right) .
\end{gathered}
$$

By removing $D$ from both sides of the above equation we get

$$
v_{R}=c+\frac{2 x}{a}\left(m_{\chi}^{2}-\omega^{2}\right) \Phi^{2}-\frac{2 y}{a} \omega^{2} \Psi^{2},
$$

where $c$ is a constant of integration. Thus we have

$$
v_{R}=c+\alpha \Phi^{2}+\beta \Psi^{2}
$$

where the constants

$$
\alpha=-\frac{2 x\left(\omega^{2}-m_{\chi}^{2}\right)}{a}, \quad \beta=-\frac{2 y \omega^{2}}{a}
$$

do not depend on $\omega$ since $v_{R}$ does not.

We now substitute the expressions for $R_{\omega}$ and $v_{R}$ into the equation that defines $v_{R}$. We have

$$
\begin{gathered}
c+\alpha \Phi^{2}+\beta \Psi^{2}=\lambda_{R}\left(\Phi^{2}+\Psi^{2}-\phi_{0}^{2}\right)+ \\
+\frac{\lambda_{R} N}{2 \pi^{2}} \int_{m_{\chi}} d \omega \omega \sqrt{\omega^{2}-m_{\chi}^{2}}\left[a-\frac{\alpha a}{2\left(\omega^{2}-m_{\chi}^{2}\right)} \Phi^{2}-\frac{\beta a}{2 \omega^{2}} \Psi^{2}+\frac{1}{4 \omega^{3}}\left(c+\alpha \Phi^{2}+\beta \Psi^{2}\right)-\frac{1}{2 \omega}\right] .
\end{gathered}
$$

From the above equation we get

$$
\begin{gathered}
\alpha=\lambda_{R}+\frac{\alpha \lambda_{R} N}{2 \pi^{2}} \int_{m_{\chi}} d \omega \omega \sqrt{\omega^{2}-m_{\chi}^{2}}\left[\frac{1}{4 \omega^{3}}-\frac{a}{2\left(\omega^{2}-m_{\chi}^{2}\right)}\right], \\
\beta=\lambda_{R}+\frac{\beta \lambda_{R} N}{2 \pi^{2}} \int_{m_{\chi}} d \omega \omega \sqrt{\omega^{2}-m_{\chi}^{2}}\left[\frac{1}{4 \omega^{3}}-\frac{a}{2 \omega^{2}}\right] .
\end{gathered}
$$

One can also extract an equation for the constant part of Eq. (5.35)

$$
c+\beta \phi_{0}^{2}=\frac{\beta \lambda_{R} N}{2 \pi^{2}} \int_{m_{\chi}} d \omega \omega \sqrt{\omega^{2}-m_{\chi}^{2}}\left[a-\frac{\beta a}{2 \omega^{2}} \phi_{0}^{2}+\frac{1}{4 \omega^{3}}\left(c+\beta \phi_{0}^{2}\right)-\frac{1}{2 \omega}\right] .
$$


We get three equations for four unknown parameters $\alpha, \beta, a$ and $c$.

To choose the correct solution to Eqs. (5.36), (5.37) and (5.38) we have to use the following physical condition. When the resolvent is expressed in terms of the physical (shifted) fields $\Phi^{a}$ and $\Psi^{\prime}=\Psi-\phi_{0}$ the constant part of the resolvent is fixed in perturbation theory to be equal to $1 /(2 \omega)$. This means that

$$
a-\frac{a \beta \phi_{0}^{2}}{2 \omega^{2}}=\frac{1}{2 \omega}
$$

and hence

$$
a=\frac{\omega}{2\left(\omega^{2}-\beta \phi_{0}^{2} / 2\right)} .
$$

We substitute this expression into Eq. (5.38) we get

$$
c=-\beta \phi_{0}^{2}
$$

For the parameters $\alpha$ and $\beta$ we have

$$
\begin{aligned}
& \alpha=\frac{\lambda_{R}}{1+\frac{\lambda_{R} N}{8 \pi^{2}} J_{1}}, \\
& \beta=\frac{\lambda_{R}}{1+\frac{\lambda_{R} N}{8 \pi^{2}} J_{2}},
\end{aligned}
$$

where

$$
\begin{gathered}
J_{1}=\int_{m_{\chi}} d \omega \sqrt{\omega^{2}-m_{\chi}^{2}}\left[\frac{\omega^{2}}{\left(\omega^{2}-\beta \phi_{0}^{2} / 2\right)\left(\omega^{2}-m_{\chi}^{2}\right)}-\frac{1}{\omega^{2}}\right], \\
J_{2}=\int_{m_{\chi}} d \omega \sqrt{\omega^{2}-m_{\chi}^{2}}\left[\frac{1}{\omega^{2}-\beta \phi_{0}^{2} / 2}-\frac{1}{\omega^{2}}\right] .
\end{gathered}
$$

It is easy to see that the parameters $\alpha$ and $\beta$ are real in contrast to the case without the spontaneous breaking of the $O(N)$-symmetry.

For the resolvent we get the following expression

$$
R_{\omega}\left[v_{R}\right]=\frac{1}{2 \omega\left(\omega^{2}-\beta \phi_{0}^{2} / 2\right)}-\frac{\alpha \omega \Phi^{2}}{4\left(\omega^{2}-m_{\phi}^{2} / 4\right)\left(\omega^{2}-m_{\chi}^{2}\right)}-\frac{\beta \Psi^{2}}{4 \omega\left(\omega^{2}-m_{\phi}^{2} / 4\right)},
$$

where we identified the physical mass of the field $\Psi^{\prime}$ with

$$
m_{\phi}^{2}=2 \beta \phi_{0}^{2}
$$

One can see that the resolvent has two poles at $\omega=m_{\chi}$ and $\omega=m_{\phi} / 2$. It can be easily checked in perturbation theory with respect to the external lines at the tree level for the resolvent that exactly these two poles appear to the lowest orders. Indeed the pole at $\omega=m_{\chi}$ corresponds to a diagram with two external legs of the $\chi$ field while the pole at $\omega=m_{\phi} / 2$ comes from the diagram with a single $\phi$ external leg. It is worth noticing that by looking at Eq. (5.46) one might think that there is a pole at $\omega=m_{\phi} / 2$ in the 
amplitude to produce two $\chi$ particles which does not of course appear in perturbation theory. It is easy to check however that such a pole is cancelled if we take into account the explicit solution for $\Phi^{a}$ and $\Psi$ which is given below. One can also see that there are no poles at $\omega=m_{\phi}$ in the resolvent. This signals the fact that the amplitudes for the production of two external $\phi$ particles are cancelled in contrast to the case without the spontaneous breaking.

Thus we get the following equations for the generating functions

$$
\begin{gathered}
\left\{D^{2}-m_{\chi}^{2}-\left(\alpha \Phi^{2}+\beta \Psi^{2}-\beta \phi_{0}^{2}\right)\right\} \Phi^{a}=0, \\
\left\{D^{2}-\left(\alpha \Phi^{2}+\beta \Psi^{2}-\beta \phi_{0}^{2}\right)\right\} \Psi=0 .
\end{gathered}
$$

These equations reduce to the classical ones (with the renormalized coupling constant $\lambda_{R}$ ) if we renormalize the fields $\Phi^{a}$ and $\Psi$ as follows

$$
\tilde{\Phi}^{a}=\sqrt{\frac{\alpha}{\lambda_{R}}} \Phi^{a}=\Phi^{a}\left(1+\frac{\lambda_{R} N}{8 \pi^{2}} J_{1}\right)^{-1 / 2}, \quad \tilde{\Psi}=\sqrt{\frac{\beta}{\lambda_{R}}} \Psi=\Psi\left(1+\frac{\lambda_{R} N}{8 \pi^{2}} J_{2}\right)^{-1 / 2} .
$$

The solution to the above equations which obeys the condition that at $\lambda_{R} \rightarrow 0$

$$
\begin{gathered}
\Phi^{a} \rightarrow m_{\chi} \xi_{1}^{a} e^{m_{\chi} \tau}, \\
\Psi \rightarrow \phi_{0}+m_{\phi} \xi_{2} e^{m_{\phi} \tau}
\end{gathered}
$$

is similar to that given in Ref. [23]:

$$
\begin{gathered}
\tilde{\Psi}=\phi_{0 R}\left(1+\frac{\tilde{z}_{2}}{2 \phi_{0 R}}+\frac{8 \lambda_{R}}{4 m_{\chi}^{2}-m_{\phi}^{2}} \tilde{z}_{1}^{2}+\frac{4 \lambda_{R}}{\phi_{0 R}} \frac{2 m_{\chi}-m_{\phi}}{\left(2 m_{\chi}+m_{\phi}\right)^{3}} \tilde{z}_{2} \tilde{z}_{1}^{2}\right) \times \\
\left(1-\frac{\tilde{z}_{2}}{2 \phi_{0 R}}-\frac{8 \lambda_{R}}{4 m_{\chi}^{2}-m_{\phi}^{2}} \tilde{z}_{1}^{2}+\frac{4 \lambda_{R}}{\phi_{0 R}} \frac{2 m_{\chi}-m_{\phi}}{\left(2 m_{\chi}+m_{\phi}\right)^{3}} \tilde{z}_{2} \tilde{z}_{1}^{2}\right)^{-1}, \\
\tilde{\Phi}^{a}=\tilde{z}_{1}^{a}\left(1-\frac{2 m_{\chi}-m_{\phi}}{2 m_{\chi}+m_{\phi}} \frac{\tilde{z}_{2}}{2 \phi_{0 R}}\right)\left(1-\frac{\tilde{z}_{2}}{2 \phi_{0 R}}-\frac{8 \lambda_{R}}{4 m_{\chi}^{2}-m_{\phi}^{2}} \tilde{z}_{1}^{2}+\frac{4 \lambda_{R}}{\phi_{0 R}} \frac{2 m_{\chi}-m_{\phi}}{\left(2 m_{\chi}+m_{\phi}\right)^{3}} \tilde{z}_{2} \tilde{z}_{1}^{2}\right)^{-1} .
\end{gathered}
$$

Here

$$
\phi_{0 R}=\phi_{0} \sqrt{\frac{\beta}{\lambda_{R}}}=\phi_{0}\left(1+\frac{\lambda_{R} N}{8 \pi^{2}} J_{2}\right)^{-1 / 2}
$$

and

$$
\begin{aligned}
& \tilde{z}_{1}^{a}=m_{\chi} \xi_{1}^{a} e^{m_{\chi} \tau}\left(1+\frac{\lambda_{R} N}{8 \pi^{2}} J_{1}\right)^{-1 / 2} \\
& \tilde{z}_{2}=m_{\phi} \xi_{2} e^{m_{\phi} \tau}\left(1+\frac{\lambda_{R} N}{8 \pi^{2}} J_{2}\right)^{-1 / 2}
\end{aligned}
$$

Thus we finally get

$$
\Psi=\phi_{0}\left(1+\frac{\tilde{z_{2}}}{2 \phi_{0 R}}+\frac{8 \lambda_{R}}{4 m_{\chi}^{2}-m_{\phi}^{2}} \tilde{z}_{1}^{2}+\frac{4 \lambda_{R}}{\phi_{0 R}} \frac{2 m_{\chi}-m_{\phi}}{\left(2 m_{\chi}+m_{\phi}\right)^{3}} \tilde{z}_{2} \tilde{z}_{1}^{2}\right) \times
$$




$$
\begin{gathered}
\left(1-\frac{\tilde{z_{2}}}{2 \phi_{0 R}}-\frac{8 \lambda_{R}}{4 m_{\chi}^{2}-m_{\phi}^{2}} \tilde{z}_{1}^{2}+\frac{4 \lambda_{R}}{\phi_{0 R}} \frac{2 m_{\chi}-m_{\phi}}{\left(2 m_{\chi}+m_{\phi}\right)^{3}} \tilde{z}_{2} \tilde{z}_{1}^{2}\right)^{-1}, \\
\Phi^{a}=z_{1}^{a}\left(1-\frac{2 m_{\chi}-m_{\phi}}{2 m_{\chi}+m_{\phi}} \frac{\tilde{z}_{2}}{2 \phi_{0 R}}\right)\left(1-\frac{\tilde{z_{2}}}{2 \phi_{0 R}}-\frac{8 \lambda_{R}}{4 m_{\chi}^{2}-m_{\phi}^{2}} \tilde{z}_{1}^{2}+\frac{4 \lambda_{R}}{\phi_{0 R}} \frac{2 m_{\chi}-m_{\phi}}{\left(2 m_{\chi}+m_{\phi}\right)^{3}} \tilde{z}_{2} \tilde{z}_{1}^{2}\right)^{-1} .
\end{gathered}
$$

We observe that this solution is real.

\section{Conclusions}

Let us briefly summarize our results. We presented an exact solution in the large- $N$ limit for the amplitudes of multiparticle production at threshold in the $\phi^{4}$ theory with the $O(N)$-symmetry which is softly broken to $O\left(N_{1}\right) \times \ldots \times O\left(N_{s}\right)\left(N=N_{1}+\ldots+N_{s}\right)$ by the mass term. We found that the effect of loops in this limit reduces to a renormalization of coupling constants and masses. Moreover as the masses $m_{i}$ of the $O\left(N_{i}\right)$-multiplets are nonequal, the amplitudes of multiparticle production become complex. The explicit form of the solution demonstrates a nullification of the multiparticle amplitudes with all legs on mass-shell. The only non-vanishing amplitudes correspond to the processes $2 \rightarrow 2$ when the incoming (outgoing) particles are identical.

We also analyzed the model of the $O(N)+$ singlet scalar particles in the case when the reflection symmetry is spontaneously broken. In the limit of large $N$ the effect of loops of the $O(N)$ field reduces to a renormalization of the coupling constants and masses of the physical particles while no imaginary part of amplitudes appears in this case. Using the exact solution for this problem, we demonstrate a nullification of amplitudes except for those with 2 incoming $O(N)$ particles and 2 outgoing $O(N)$ particles or 1 outgoing physical singlet particle.

Some problems remain unsolved. As it has been demonstrated in Ref. [24], the nullification of the tree level amplitudes for the model of two scalar fields with further restrictions on kinematics, when one particle, say $m_{2}$, can decay at rest into the others with the mass $m_{1}$, is due to a symmetry of the classical Lagrangian where the dependence of the fields on the spatial 3D coordinate is discarded. It is straightforward to check that such a symmetry exists at the tree level in the $O\left(N_{1}\right) \times O\left(N_{2}\right)$ model as well. Indeed the Lagrangian (2.1) is invariant (up to terms which are total derivatives) for the fields independent of the spatial coordinates under the following transformations

$$
\begin{gathered}
\delta \chi^{a}=\epsilon \lambda \phi^{\alpha}\left(\dot{\chi}^{a} \phi^{\alpha}-\dot{\phi}^{\alpha} \chi^{a}\right), \\
\delta \phi^{\alpha}=\epsilon\left[-\lambda \chi^{a}\left(\dot{\chi}^{a} \phi^{\alpha}-\dot{\phi}^{\alpha} \chi^{a}\right)+\frac{1}{2}\left(m_{1}^{2}-m_{2}^{2}\right) \dot{\phi}^{\alpha}\right],
\end{gathered}
$$

where $\dot{\phi}$ stands for $d \phi / d t$ and $\epsilon$ is a constant parameter of the variation. Following Ref. 24] one can see that the non-vanishing amplitudes come from the resonance terms 
which appear in the iteration procedure for solving the classical equations of motion with the boundary conditions at $\lambda \rightarrow 0$ :

$$
\chi \rightarrow \xi_{1}^{a} e^{-i m_{1} t}, \quad \phi \rightarrow \xi_{2}^{\alpha} e^{-i m_{2} t}
$$

The explicit form of our solution shows that such a symmetry survives at the multi-loop level (at large $N$ ) since the only effect of multi-loop corrections reduces to a renormalization of the coupling constants and masses. Obviously we come to the same conclusion in the case of a spontaneously broken reflection symmetry in the model of the $O(N)+$ singlet scalar particles. An interesting question about this symmetry is whether it can be extended to arbitrary $m_{1}$ and $m_{2}$ when kinematics requires nonvanishing spatial momenta of incoming particles.

As it is already mentioned in Subsect. 3.2, our results for the multiparticle amplitudes are applicable when the number of produced particles is much less than $\sqrt{N}$. They nicely show how the loop effects renormalize tree amplitudes but do not take into account diagrams with rescatterings in the final state which are expected to restore unitarity. There exist at the moment two examples of such calculations: 1) a semiclassical calculation [22] for the $N=1$ case with a spontaneously broken reflection symmetry, 2) models with any $N$ in $2+1$ dimensions which are solved [27 by summing infrared logarithms with the aid of renormalization group. It would be interesting to develop a technique based on the Schwinger-Dyson equations which would be applicable for the case when the number of produced particles is of order $\sqrt{N}$. We hope that the operator formulation of the Schwinger-Dyson equations for the multiparticle amplitudes which is proposed in this paper may be useful for this purpose.

\section{Acknowledgments}

M.A. and Yu.M. were sponsored, in part, by the Danish Natural Science Research Council. A.J. acknowledges the NBI high energy group for its hospitality. His research was supported in part by a NATO grant GRG 930395 . 


\section{References}

[1] J.M. Cornwall, Phys. Lett. B243 (1990) 271.

[2] H. Goldberg, Phys. Lett. B246 (1990) 445.

[3] M.B. Voloshin, Phys. Rev. D43 (1991) 1726.

[4] J.M. Cornwall and G. Tiktopoulos, Phys. Rev. D45 (1992) 2105.

[5] H. Goldberg and M.T. Vaughn, Phys. Rev. Lett. 66 (1991) 1267.

[6] M.B. Voloshin, Nucl. Phys. B383 (1992) 233.

[7] E.N. Argyres, R. Kleiss and C.G. Papadopoulos, Nucl. Phys. B391 (1993) 42.

[8] E.N. Argyres, R. Kleiss and C.G. Papadopoulos, Nucl. Phys. B391 (1993) 57.

[9] M.B. Voloshin, Phys. Lett. B293 (1992) 389.

[10] L.S. Brown, Phys. Rev. D46 (1992) R4125.

[11] E.N. Argyres, R. Kleiss and C.G. Papadopoulos, Phys. Lett. B296 (1992) 139.

[12] M.B. Voloshin, Phys. Rev. D47 (1993) R357; 1712.

[13] B.H. Smith, Phys. Rev. D47 (1993) 3518.

[14] M.B. Voloshin, Phys. Rev. D47 (1993) 2573.

[15] B.H. Smith, Phys. Rev. D47 (1993) 3521.

[16] M.B. Voloshin, Phys. Rev. D47 (1993) 3525.

[17] L.S. Brown and C. Zhai, Phys. Rev. D47 (1993) 5526.

[18] E.N. Argyres, R. Kleiss and C.G. Papadopoulos, Phys. Lett. B302 (1993) 70.

[19] B.H. Smith, Phys. Rev. D47 (1993) 5531.

[20] E.N. Argyres, R. Kleiss and C.G. Papadopoulos, Phys. Lett. B308 (1993) 292.

[21] E.N. Argyres, R. Kleiss and C.G. Papadopoulos, Phys. Lett. B308 (1993) 315.

[22] A.S. Gorsky and M.B. Voloshin, Phys. Rev. D48 (1993) 3843.

[23] M.V. Libanov, V.A. Rubakov and S.V. Troitsky, Nucl. Phys. B412 (1994) 607.

[24] M.V. Libanov, V.A. Rubakov and S.V. Troitsky, Phys. Lett. B318 (1993) 134.

[25] B.H. Smith, Phys. Rev. D49 (1994) 1081.

[26] Yu. Makeenko, Exact multiparticle amplitudes at threshold in large- $N$ component $\phi^{4}$ theory, NBI-HE-94-25, hep-ph/9404312 (April, 1994).

[27] V.A. Rubakov and D.T. Son, Renormalization group for multiparticle production in $(2+1)$ dimensions around the threshold, INR preprint, hep-ph/9406362 (July, 1994).

[28] K.G. Wilson, Phys. Rev. D7 (1973) 2911.

[29] Yu.M. Makeenko, Large N, in Gauge Theories of the Eighties, eds. R. Raitio and J. Lindfors, Lecture Notes in Physics 181, Springer-Verlag, 1983, p. 67.

[30] I.M. Gelfand and L.A. Dikiǔ, Russ. Math. Surv. 30 (1975) 77. 